\title{
Compensation of head-on beam-beam induced resonance driving terms and tune spread in the Relativistic Heavy Ion Collider
}

\author{
W. Fischer, X. Gu, K. A. Drees, C. Liu, Y. Luo, A. Marusic, R. Michnoff, T. A. Miller, M. Minty, \\ C. Montag, A. I. Pikin, G. Robert-Demolaize, V. Schoefer, and P. Thieberger \\ Brookhaven National Laboratory, Upton, New York 11973, USA
}

S. M. White

European Synchrotron Radiation Facility, BP 220, 38043 Grenoble Cedex, France

(Received 12 February 2017; published 13 September 2017)

\begin{abstract}
A head-on beam-beam compensation scheme was implemented for operation in the Relativistic Heavy Ion Collider (RHIC) at Brookhaven National Laboratory [Phys. Rev. Lett. 115, 264801 (2015)]. The compensation consists of electron lenses for the reduction of the beam-beam induced tune spread, and a lattice for the minimization of beam-beam generated resonance driving terms. We describe the implementations of the lattice and electron lenses, and report on measurements of lattice properties and the effect of the electron lenses on the hadron beam.
\end{abstract}

DOI: 10.1103/PhysRevAccelBeams.20.091001

\section{INTRODUCTION}

In Ref. [1] the implementation of operational head-on beam-beam compensation in the Relativistic Heavy Ion Collider (RHIC) is summarized. The implemented compensation scheme consists of electron lenses for the reduction of the beam-beam induced tune spread, and a lattice for the minimization of resonance driving terms. Here we present in more detail the design and verification of the lattice, and measurements of the effects of the electron lenses on the proton and other ion beams. Reference [1] also provides background information on the only previous attempt at operational beam-beam compensation in DCI [2,3] as well as the development of the electron lens technology and use in the Tevatron [4-9]. Details of the RHIC electron lens technology are given in Refs. [10-14].

For equal normalized rms emittances $\epsilon_{n}$ in both transverse planes, the beam-beam parameter for proton-proton collisions is $\xi_{p}=-\left(r_{p} N_{p}\right) /\left(4 \pi \epsilon_{n}\right)$, where $r_{p}$ is the classical proton radius, $N_{p}$ the bunch intensity, and $\epsilon_{n}=$ $\left(\beta_{p} \gamma_{p}\right) \sigma_{p}^{2} / \beta .\left(\beta_{p} \gamma_{p}\right)$ are the relativistic factors of the proton beam, $\sigma_{p}$ is the transverse rms beam size and $\beta$ the lattice function. We recall the luminosity formula for round Gaussian and equal beams at the interaction point (IP), as is the case in RHIC [1,15]:

\footnotetext{
*Wolfram.Fischer@bnl.gov
}

Published by the American Physical Society under the terms of the Creative Commons Attribution 4.0 International license. Further distribution of this work must maintain attribution to the author(s) and the published article's title, journal citation, and DOI.

$$
\mathcal{L}=\frac{f_{c}}{4 \pi} \frac{N_{p}^{2}}{\sigma_{p}^{* 2}} H=\frac{4 \pi f_{c}}{r_{p}^{2}} \frac{\left(\beta_{p} \gamma_{p}\right) \epsilon_{n}}{\beta^{*}} H \xi_{p}^{2}
$$

where $f_{c}$ is the collision frequency, $\sigma_{p}^{*}$ the beam size at the IP, $H$ a geometric factor $\leq 1$ that accounts for the hourglass effect and crossing angles, and $\beta^{*}$ the lattice function at the IP. Due to the quadratic dependence of the luminosity $\mathcal{L}$ on the beam-beam parameter $\xi_{p}$, a relative increase in $\xi_{p}$ implemented through an increase in $N_{p}$ at constant $\epsilon_{n}$ translates into more than twice the relative increase in $\mathcal{L}$. In RHIC head-on beam-beam compensation is implemented in a single turn (Fig. 1) for one of the two head-on beambeam interactions, and we repeat the compensation principle presented in Refs. [1,16,17].

We are using transverse phase space coordinates $\left(r, r^{\prime}\right)$ with $r^{\prime}=\frac{d r}{d s}, s$ being the path length. The transverse kick $\Delta r_{p p}^{\prime}$ that a particle receives when passing through the other beam is reversed in the same turn when the particle
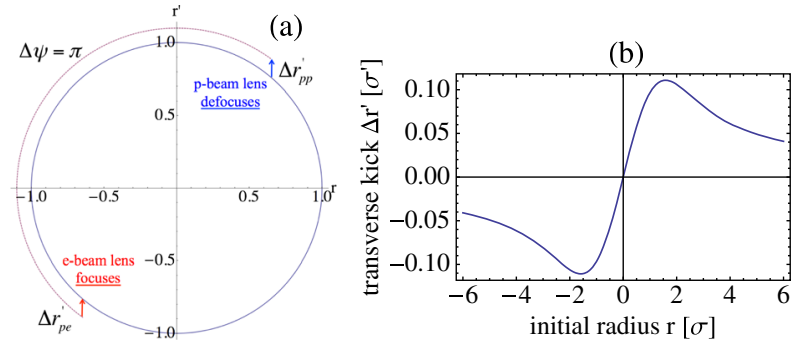

FIG. 1. Reproduced from Ref. [1]. (a) Head-on beam-beam compensation in a phase space view. A defocusing kick $\Delta r_{p p}^{\prime}$ a proton receives from the other proton beam is reversed by a focusing kick $\Delta r_{p e}^{\prime}$ from the electron lens after a phase advance $\pi$. (b) Amplitude dependence of the beam-beam kick $\Delta r^{\prime}$ on the radius $r$ [18-20]. 
passes through a correction element and receives the kick $\Delta r_{p e}^{\prime}$. Two conditions need to be fulfilled for exact compensation at all amplitudes $r$ : (i) The correction element is placed at a phase advance of $k \pi, k$ being an integer, after the beam-beam interaction in order to minimize the beam-beam resonance driving terms; (ii) The amplitude dependence of the correction kick $\Delta r_{p e}^{\prime}(r)$ is the same as for the beam-beam interaction, $\Delta r_{p p}^{\prime}(r)$, in order to reduce the beam-beam induced tune spread.

The location of the beam-beam interactions and electron lenses, one in each ring, is shown in Fig. 1. In RHIC only one of the two beam-beam interactions is compensated. Condition (i) is implemented through lattice design with a phase advance of $k \pi$ between the beam-beam interaction at IP8 and the electron lenses near IP10 in both rings (Sec. III).

Condition (ii) requires an electron beam with a Gaussian transverse profile since the proton beam also has a Gaussian transverse profile [14], an electron beam size $\sigma_{e}$ in the lenses matched to the proton beam size $\sigma_{p}$

$$
\sigma_{e}=\sigma_{p}=\sqrt{\frac{\epsilon_{n} \beta_{e l}}{\beta_{p} \gamma_{p}}}
$$

where $\beta_{e l}$ is the lattice function at the location of the electron lens, and an electron beam current matched to the proton bunch intensity

$$
I_{e}=\left(\frac{N_{p}}{L_{e}}\right) \frac{e \beta_{e} c}{1+\beta_{e}}
$$

where $\beta_{e}$ is the relativistic factor of the electrons in the lens and $L_{e}$ the magnetic length of the lens. For Eq. (3) it is assumed that the electrons and protons move in opposite directions and that the proton beam is ultrarelativisitic with $\beta_{p} \approx 1$. The more general case is presented, e.g., in Refs. $[9,21]$. The beam-beam parameters from the protonproton and proton-electron collisions with $\xi_{p}=-\xi_{e}$ are then

$$
\begin{aligned}
& \xi_{p}=-\frac{r_{p}}{4 \pi} \frac{\beta^{*}}{\left(\beta_{p} \gamma_{p}\right)} \frac{N_{p}}{\sigma_{p}^{* 2}} \text { and } \\
& \xi_{e}=+\frac{r_{p}}{4 \pi} \frac{\beta_{e l}}{\left(\beta_{p} \gamma_{p}\right)} \frac{\left(I_{e} L_{e}\right)}{\sigma_{e}^{2}} \frac{1+\beta_{e}}{e c \beta_{e}} .
\end{aligned}
$$

The operational implementation of the compensation principle requires an understanding of the tolerable deviations from the ideal case described above, and technical implementations within the allowable tolerances $[16,17]$. The main parameters of the RHIC electron lenses are shown in Table I, and a detailed description is provided in Ref. [14].
TABLE I. Typical electron lens parameters for 2015 (100 GeV proton energy) and design values (for up to $255 \mathrm{GeV}$ ) [1].

\begin{tabular}{lccc}
\hline \hline quantity & unit & 2015 value & design value \\
\hline distance of center from IP10 & $\mathrm{m}$ & \multicolumn{2}{c}{$-3.3-$} \\
magnetic length $L_{e}$ & $\mathrm{~m}$ & \multicolumn{2}{c}{$-2.4-$} \\
gun solenoid field $B_{g}$ & $\mathrm{~T}$ & 0.31 & $\leq 0.69$ \\
main solenoid field $B_{m}$ & $\mathrm{~T}$ & 5.0 & $2-6$ \\
cathode radius $(2.7 \sigma)$ & $\mathrm{mm}$ & 7.5 & $4.1,7.5$ \\
rms beam size in main & $\mu \mathrm{m}$ & 650 & $\geq 300$ \\
$\quad$ & & & \\
solenoid $\sigma_{e}$ & $\mathrm{keV}$ & 5.0 & $\leq 10$ \\
kinetic energy $E_{e}$ & $\ldots$ & 0.14 & $\leq 0.2$ \\
relativistic factor $\beta_{e}$ & $\mathrm{~mA}$ & 600 & $\leq 1000$ \\
electron beam current $I_{e}$ & 0.001 & +10 & $\leq+15$ \\
beam-beam parameter & & & \\
$\quad$ from lens $\xi_{e}$ & & & \\
\hline \hline
\end{tabular}

\section{POLARIZED PROTON OPERATION IN 2015}

The head-on beam-beam effect limited the achievable luminosity in polarized proton operation in RHIC [22]. Figure 3 shows that in 2012 the initial luminosity increased with the bunch intensity but the average store luminosity did not, due to the enhanced beam-beam effect. The figure also shows that in 2015 both the peak and average luminosity increased significantly due to the implemented head-on beam-beam compensation scheme, consisting of a new lattice and the electron lenses [23].

Table II [1] shows the main operating parameters in 2012 and 2015, and parameters for tests for maximum $\left|\xi_{p}\right|$ values with and without electron lenses in 2015. The operational values for $\mathcal{L}_{\text {avg }}$ show an increase in the average store luminosity of $91 \%$, almost a factor of 2, from 2012 to 2015. To obtain an estimate of the luminosity gain from the lattice alone we take the beam-beam parameter achieved in tests without the electron lens $\left(\xi_{p}=-0.0091\right.$, Table II) and reduce the bunch intensity in the 2015 operational

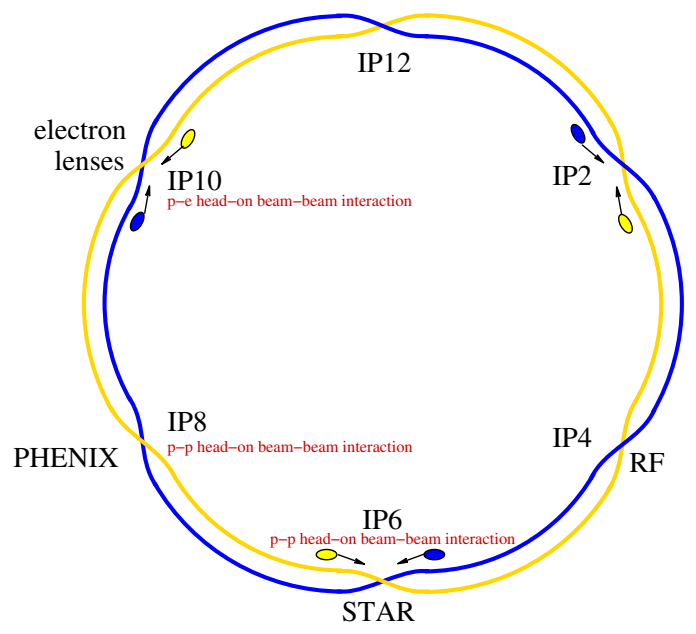

FIG. 2. General layout of RHIC with locations of the head-on beam-beam interactions and electron lenses [1]. 


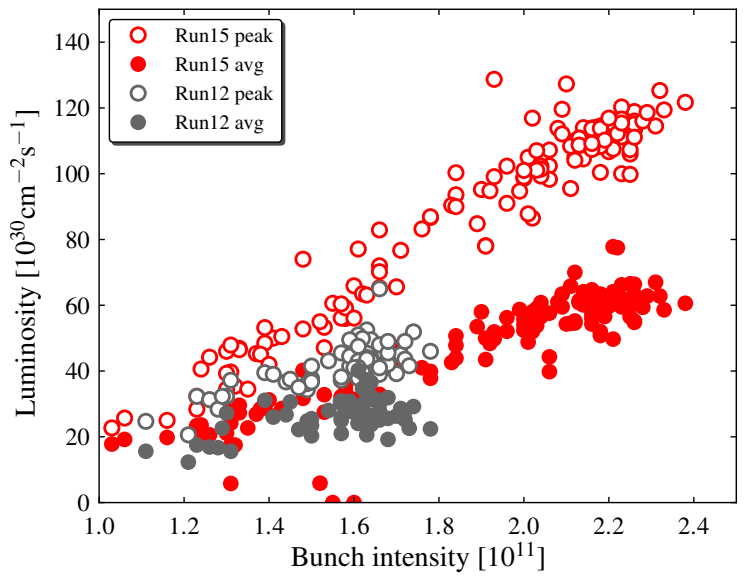

FIG. 3. Peak and average store luminosity in polarized proton operation at $100 \mathrm{GeV}$ beam energy in 2012 and 2015.

parameter set to $2.1 \times 10^{11}$ to obtain this beam-beam parameter. Further assuming an unchanged ratio of peak and average luminosity, this yields a calculated average luminosity of $\mathcal{L}_{\text {avg }}=55 \times 10^{30} \mathrm{~cm}^{-2} \mathrm{~s}^{-1}$, or a $67 \%$ luminosity gain over the 2012 value. The remainder can then be attributed to the electron lenses. Thus, approximately $2 / 3$ of the luminosity gain can be attributed to the lattice alone, and $1 / 3$ to the lattice in conjunction with the electron lenses.

In operation the electron lenses were used in the following way (Fig. 4): Both beams are accelerated to the store energy, and at time " 1 " the electron lenses are turned on with current, before the proton beams are in collision. The proton beams are transversely separated in the experimental IPs, and the electron and proton beams in the lens are transversely separated in the electron lenses. At time " 2 " the beams are brought into collision in the PHENIX experiment by removing the transverse separation. After that the collimators, in the same interaction region (IR) as PHENIX, are set to the store values. At time " 3 " the beams are brought into collision at the STAR experiments and the electron lenses simultaneously by removing the transverse separation, while the electron lenses prevent large beam losses and/or emittance growth for the highest beam-beam parameters $\xi_{p}$. At time " 4 " the electron lenses are gradually ramped down when the lattice can support the beam-beam parameter in order to avoid any detrimental effects the electron lenses have on the beam loss rates, emittance growth and background rates. These effects are presented in Sec. VI. During the ramp-down the lattice tunes are adjusted to account for the tune change due to the changed electron lens current.

The electron lenses were on for typically $1-1.5 \mathrm{~h}$ at the beginning of the store, and are only needed as long as the lattice cannot support the beam-beam parameter $\xi_{p}$ [1]. The electron beam size was set to exceed the proton beam size [16], and the electron beam current not to exceed the strength of one beam-beam interaction. All these measures were taken to minimize the possibly detrimental effects of the electron lenses (see Sec. VI).

Depolarization effects from the beam-beam interaction were not expected $[24,25]$, nor were any observed to date except via changes of the tune and tune distribution.

TABLE II. Main parameters for polarized proton operation at $100 \mathrm{GeV}$ beam energy in 2012 (without) and 2015 (with head-on beam-beam compensation), and conditions for the maximum beam-beam parameters achieved in operation and tests in 2015 without and with electron lenses [1].

\begin{tabular}{|c|c|c|c|c|c|c|}
\hline \multirow[b]{3}{*}{ quantity } & \multirow[b]{3}{*}{ unit } & & & \multicolumn{3}{|c|}{ tests for $\max \left|\xi_{p}\right|$} \\
\hline & & \multicolumn{2}{|c|}{$\begin{array}{c}\text { operations } \\
\text { (avg. over } 10 \text { best stores) }\end{array}$} & $\begin{array}{l}\text { without } \\
\text { e-lens }\end{array}$ & $\begin{array}{l}\text { with } \\
\text { e-lens }\end{array}$ & $\begin{array}{l}\text { with } \\
\text { e-lens }\end{array}$ \\
\hline & & 2012 & 2015 & \multicolumn{3}{|c|}{$-2015-$} \\
\hline bunch intensity $N_{p}$ & $10^{11}$ & 1.6 & 2.25 & 2.6 & 2.15 & 2.0 \\
\hline no of bunches $k_{b}$ & $\cdots$ & 109 & 111 & 48 & 111 & 30 \\
\hline$\beta_{x, y}^{*}$ at IP6, IP8 $(\mathrm{p}+\mathrm{p})$ & $\mathrm{m}$ & 0.85 & 0.85 & & -0.85 & \\
\hline$\beta_{x, y}^{*}$ at e-lens $(\mathrm{p}+\mathrm{e})$ & $\mathrm{m}$ & 10.5 & 15.0 & & -15.0 & \\
\hline lattice tunes $\left(Q_{x}, Q_{y}\right)$ & $\cdots$ & \multicolumn{2}{|c|}{$(0.695,0.685)$} & \multicolumn{3}{|c|}{$-(0.695,0.685)-$} \\
\hline rms emittance $\epsilon_{n}$ & $\mu \mathrm{m}$ & 3.3 & 2.8 & 3.5 & 2.4 & 1.9 \\
\hline rms beam size IP6/8 $\sigma_{p}^{*}$ & $\mu \mathrm{m}$ & 165 & 150 & 170 & 150 & 125 \\
\hline rms beam size e-lens $\sigma_{p}$ & $\mu \mathrm{m}$ & - & 630 & 700 & 645 & 520 \\
\hline rms bunch length $\sigma_{s}$ & $\mathrm{~m}$ & 0.63 & 0.70 & 0.77 & 0.70 & 0.56 \\
\hline rms momentum spread $\sigma_{\delta}$ & 0.001 & 0.32 & 0.42 & 0.46 & 0.52 & 0.31 \\
\hline hourglass factor $H$ & $\ldots$ & 0.74 & 0.75 & 0.78 & 0.81 & 0.86 \\
\hline beam-beam param. $\xi_{p} / \mathrm{IP}$ & 0.001 & -5.8 & -9.7 & -9.1 & -10.9 & -12.6 \\
\hline \# of beam-beam IPs & $\ldots$ & 2 & $2+1^{*}$ & 2 & $2+1^{*}$ & $2+1^{*}$ \\
\hline luminosity $\mathcal{L}_{\text {peak }}$ & $10^{30} \mathrm{~cm}^{-2} \mathrm{~s}^{-1}$ & 46 & 115 & 72 & 115 & 40 \\
\hline luminosity $\mathcal{L}_{\text {avg }}$ & $10^{30} \mathrm{~cm}^{-2} \mathrm{~s}^{-1}$ & 33 & 63 & - & - & - \\
\hline
\end{tabular}

${ }^{\mathrm{a}}$ One $\mathrm{p}+\mathrm{p}$ collision in IP6 and IP8, and a compensating $\mathrm{p}+\mathrm{e}$ collision in IR10. 


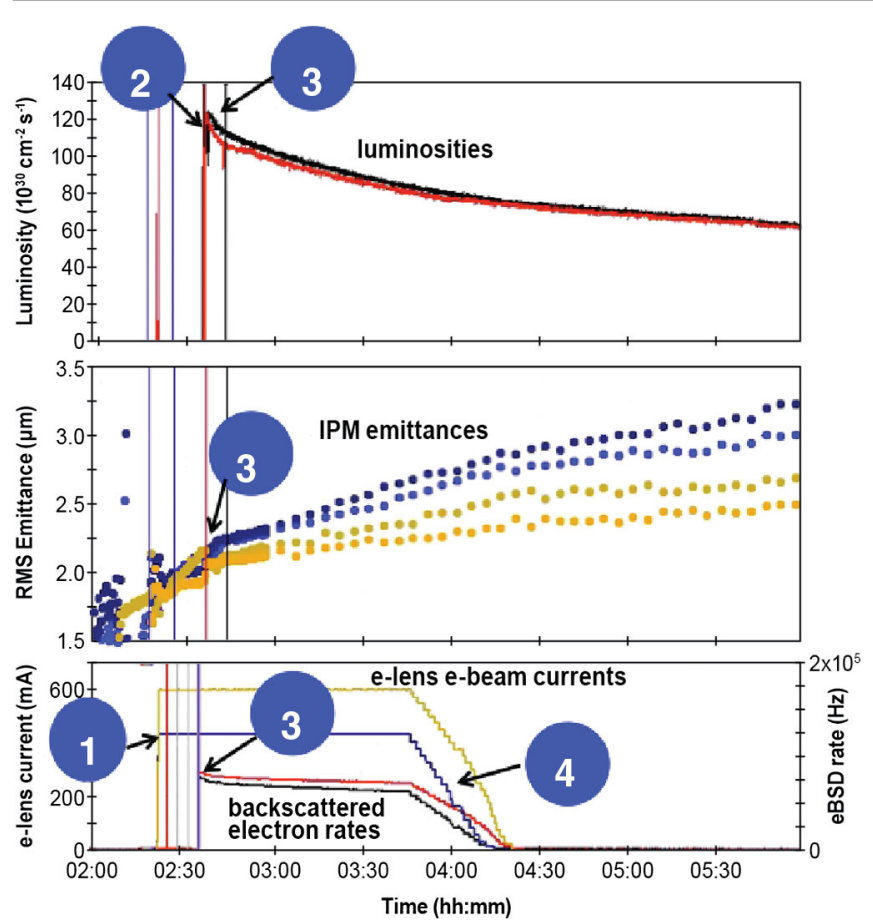

FIG. 4. Operational use of the electron lenses in 2015. The top plot shows the luminosities of both experiments, the middle plot the rms emittances as measured by the IPMs, and the bottom plot the electron beam current of both lenses (left scale) as well as the signal from the backscattered electrons (eBSD, right scale). The numbers " 1 " through " 4 " indicate times for certain events that are explained in the text.

The absolute polarization is measured in stores with an atomic polarized hydrogen jet [26,27], and delivers the polarization averaged over the intensity distribution. In 2012, without beam-beam compensation, the polarization averaged over all stores was $61.8 \%$ (Blue) and $56.6 \%$ (Yellow) $[28,29]$, with only vertically polarized beams colliding. In 2015, with beam-beam compensation, the polarization was $53.0 \%$ (Blue) and $57.4 \%$ (Yellow) but only a part of the run was with vertical polarization at the IPs. For the part with vertical polarization at the IPs the polarization was $56.2 \%$ (Blue) and $58.5 \%$ (Yellow). The relative statistical error of the polarization numbers is approximately $1 \%$ [23,29]. The measured polarization values are consistent with the expectation that the beambeam interactions, including the electron lenses, have a negligible effect on the polarization.

\section{LATTICE DESIGN AND VERIFICATION}

As discussed in Sec. I the electron lenses have to be placed at a phase advance of $k \pi, k$ being an integer, from the beam-beam interaction that is to be compensated. In RHIC only one of the two head-on beam-beam interactions is compensated and the phase advance is constrained between IP8 and the electron lenses near IP10 (Fig. 2).
Phase errors enhance the beam-beam generated resonance driving terms [16].

In addition, a transversely large proton beam at the location of the electron lens makes alignment of the two beams easier, and suppresses instabilities driven by the electron-proton beam interaction in the electron lens.

When a proton bunch interacts with the electron beam it will drive Larmor oscillations of the electrons along the interaction region resulting in an $s$-dependent kick onto the proton bunch. This can be interpreted as an electron lens impedance, which may be comparable to or larger than the machine impedance. Its strength depends on the electron lens parameters and under certain conditions can lead to a transverse mode coupling instability (TMCI). Under the approximation of a linear beam-beam force the instability threshold for the electron lens solenoid field $B_{\text {th }}$ can be expressed as [30]

$$
B_{\mathrm{th}}=1.3 \frac{e N_{p} \xi_{e}}{\rho^{2} \sqrt{\Delta Q Q_{s}}} .
$$

where $\rho$ is the radius of the proton or electron beam, which are approximately equal [cf. Eq. (2)] and $\rho \approx \sqrt{2} \sigma$ for Gaussian distribution. $\Delta Q$ is the separation between horizontal and vertical tunes and $Q_{s}$ is the synchrotron tune. Increasing the beam size at the electron lens by increasing the $\beta$-function mitigates this instability. Numerical simulations showed that a transverse bunchby-bunch damper could also cure these instabilities [31]. An experiment was performed with Au beam in 2014, in which the solenoid main field was lowered to $1.5 \mathrm{~T}$, which still allowed for electron beam propagation through the lens, and for which simulations showed unstable behavior. No instability was observed in the experiment [32]. A damper design exists and tests were made in 2015. So far, a transverse damper was not necessary in operation.

All RHIC lattices for proton-proton collisions also must minimize effects that depolarize the beam on the ramp or during store. This was studied in detail for a future lattice for $255 \mathrm{GeV}$ beam energy [33].

\section{A. Achromatic telescope squeezing (ATS) [34] optics for RHIC}

Previous studies showed that as long as the lattice tune is not in the proximity of a low order betatron resonance, the colliding proton beam lifetime is limited by the beam-beam interaction and the off-momentum dynamic aperture [22]. In the process of developing a new lattice, a dedicated effort was made to also minimize the nonlinear chromaticity in order to provide for an increased off-momentum dynamic aperture [35].

For nonzero chromaticity, off-momentum particles will experience tune shifts and optics distortion with respect to the on-momentum reference particle. The resulting 
momentum dependent tunes and $\beta$-functions can be expressed as:

$$
\begin{gathered}
Q\left(\delta_{p}\right)=Q_{0}+Q^{\prime} \delta_{p}+\frac{1}{2} Q^{\prime \prime} \delta_{p}^{2}+\frac{1}{6} Q^{\prime \prime \prime} \delta_{p}^{3}+\cdots, \\
\beta\left(\delta_{p}\right)=\beta_{0}+\beta^{\prime} \delta_{p}+\frac{1}{2} \beta^{\prime \prime} \delta_{p}^{2}+\frac{1}{6} \beta^{\prime \prime \prime} \delta_{p}^{3}+\cdots,
\end{gathered}
$$

where $Q_{0}$ and $\beta_{0}$ are the unperturbed tune and $\beta$-function. The first and second order chromaticities can be expressed as:

$$
\begin{aligned}
& Q_{x, y}^{\prime}=-\frac{1}{4 \pi} \int_{0}^{C} K_{x, y}(s) \beta_{x, y}(s) d s, \\
& Q_{x, y}^{\prime \prime}=-\frac{1}{4 \pi} \int_{0}^{C} K_{x, y}(s) \beta_{x, y}^{\prime}(s) d s,
\end{aligned}
$$

with

$$
\begin{aligned}
& K_{x}=+K_{1}(s)-K_{2}(s) D_{x}(s), \\
& K_{y}=-K_{1}(s)+K_{2}(s) D_{x}(s),
\end{aligned}
$$

where $K_{1}$ is the quadrupole strength, $K_{2}$ the sextupole strength and $D$ the dispersion. Here, we have neglected the contribution from bending magnets. The off-momentum $\beta$-function $\beta^{\prime}$ is given by:

$$
\begin{aligned}
\beta_{x, y}^{\prime}\left(s_{1}\right)= & \frac{\beta_{x, y}\left(s_{1}\right)}{2 \sin \left(2 \pi Q_{x, y}\right)} \int_{0}^{C} K_{x, y}(s) \beta_{x, y}(s) \\
& \times \cos \left(2\left|\phi_{x, y}(s)-\phi_{x, y}\left(s_{1}\right)\right|-2 \pi Q_{x, y}\right) d s .
\end{aligned}
$$

From these equations one can see that a proper arrangement of chromatic sextupoles would allow us to correct both the linear and nonlinear chromaticities. This topic has been described in the literature (see, for example, Ref. [34]) and will not be discussed further here.

For a squeezed optics, the $\beta$-functions at the final focusing quadrupoles are significantly increased and give rise to chromatic aberrations, which may be difficult to correct. An option for a passive correction of these aberrations is the ATS optics developed at CERN for the HL-LHC upgrade $[34,36]$. It uses a $\beta$-beat wave propagating through the arcs and low- $\beta$ insertions to further reduce the $\beta$-function at the IP without changing the chromatic properties of the lattice. As long as the presqueeze optics (before the wave is applied) is properly corrected, or naturally features low non-linear chromaticity, further squeezing using the $\beta$-beat wave will not degrade the chromaticity.

The target $\beta^{*}$ at the two experiments PHENIX and STAR for the electron lens lattice at $100 \mathrm{GeV}$ is $0.85 \mathrm{~m}$ allows for a lattice design with a naturally low nonlinear chromaticity. Another advantage of this lattice is that it relieves some of the strength of the low- $\beta$ insertions quadrupoles which in

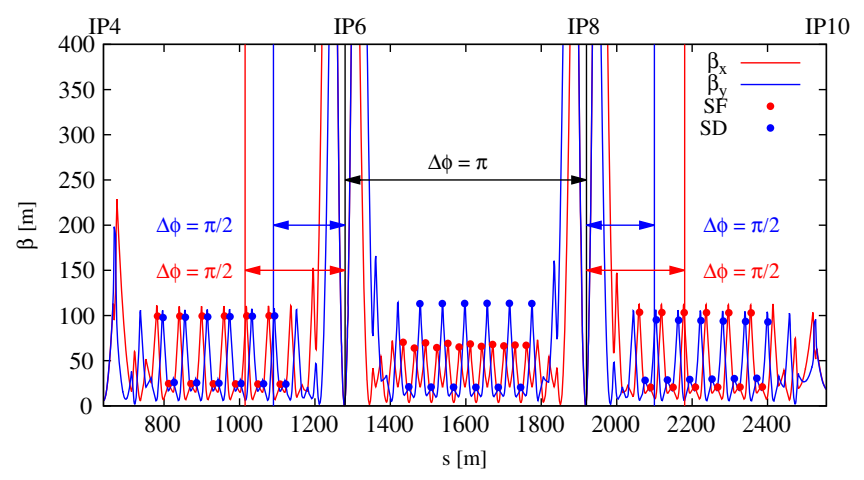

FIG. 5. RHIC Blue ring ATS lattice for $100 \mathrm{GeV}$ protons. Only a fraction of the $3.8 \mathrm{~km}$ circumference is shown.

turn can be used to match the phase advance to the electron lens. In order to achieve the ideal ATS squeeze the following constraints have to be fulfilled: (i) In RHIC the two low- $\beta$ insertions are in neighboring interaction regions (different from the LHC) and the $\beta$-beat wave will therefore propagate through both insertions before being damped down again. In order to squeeze both IRs at the same time the phase advance between the two IPs has to be $k \pi, k$ being an integer. (ii) In order to ensure proper chromaticity correction a $90^{\circ}$ FODO (a cell consisting of four optical elements in the sequence: focusing, not focusing, defocusing, not focusing) lattice is required and a phase advance of $\pi / 2$ is required between the IP and one of the sextupole families in both planes.

These constraints have to be fulfilled before the $\beta$-beat wave is applied, i.e. for the pre-squeeze optics. The $90^{\circ}$ FODO lattice required an increase in the integer tune by one unit in both planes and as a consequence a new injection optics was produced involving new power supplies. Details of the design study can be found in [35].

Figure 5 shows the ATS lattice for the RHIC Blue ring. The $\beta$-beat wave is launched in IR4 and closed in IR10. The phase advance and final $\beta$-function at the electron lenses are set during the ATS squeeze. Using this method it was possible to exactly match the phase advance to $k \pi$ and increase the $\beta$-function at the electron lens to $15 \mathrm{~m}$. The Yellow ring features similar properties. As shown in Fig. 6 the nonlinear chromaticity of the 2015 ATS lattices is equal to or smaller than the one of the 2012 lattices for all planes but the Blue vertical one. For the Blue vertical plane the tune deviation increases visibly for a negative momentum deviation $\delta p / p$ but its value is still smaller than for the 2012 lattice in all other planes.

\section{B. Long bunches}

With a proton bunch length $\sigma_{z}$ comparable to or larger than $\beta^{*}, \sigma_{z} \gtrsim \beta^{*}$, the phase advance during the passage of a proton through the other beam changes, and the phase advance condition $k \pi$ between the $\mathrm{p}+\mathrm{p}$ and $\mathrm{p}+\mathrm{e}$ interactions is only approximately fulfilled [21]. 

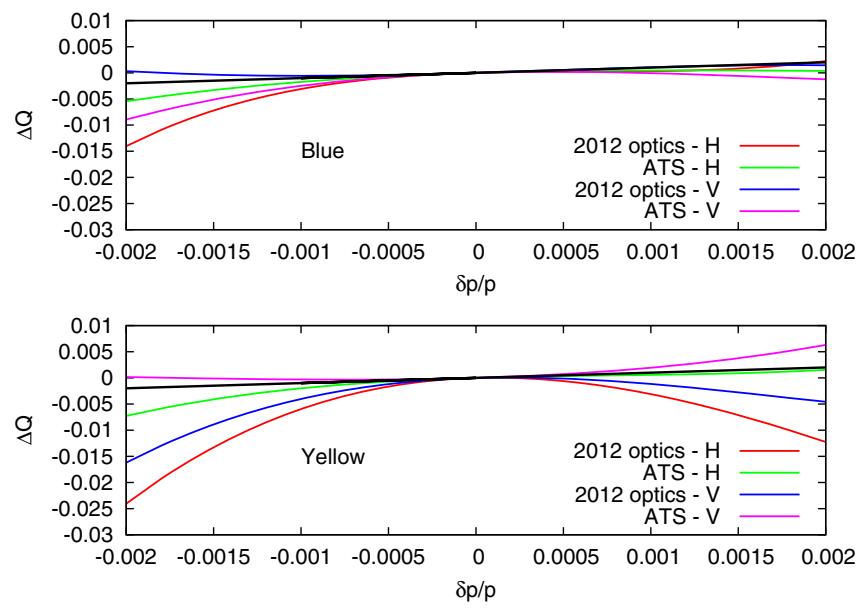

FIG. 6. Blue (top) and Yellow (bottom) non-linear tune dependence on the momentum deviation $\delta p / p$ for the 2012 lattice and the 2015 ATS optics. The black line in both plots corresponds to a linear chromaticity $Q^{\prime}=+1$.

Figure 7 shows this effect for $\sigma_{z}=0.7 \mathrm{~m}$ and $\beta^{*}=$ $0.85 \mathrm{~m}$, the values in 2015 (Table II). The phase space vector $\vec{R}=\left(r, r^{\prime}\right)$ at IP10 is plotted as a function of the initial $\left(r_{i}, r_{i}^{\prime}\right)$ at IP8, where the particle is launched. At IP10 the particle has undergone a $\mathrm{p}+\mathrm{p}$ interaction at IP8 and a $\mathrm{p}+\mathrm{e}$ interaction near IP10. Figure 7(a) shows $\vec{R}$ without head-on beam-beam compensation, and part (b) with compensation using a matched electron beam size and current. Part (c) shows the situation with an additional linear phase advance error $\psi$ between the $\mathrm{p}+\mathrm{p}$ and $\mathrm{p}+\mathrm{e}$ interactions of $5 \mathrm{deg}$.

For complete compensation one would have $R=$ $|\vec{R}|=0$ for all $\left(r_{i}, r_{i}^{\prime}\right)$ in part (b) and (c). The figures show that the largest deviations are reached for $r_{i} \approx 1 \sigma$ and large $r_{i}^{\prime}$. Deviations from complete compensation are also created by particles arriving early or late relative to the bunch center of the opposing beam, mismatches of the electron beam size to the proton beam size, or mismatches of the electron beam current to the proton bunch intensity [21]. The full effect of these deviations can only be assessed with long-term simulations (Sec. III C).

\section{Dynamic aperture studies}

Dynamic aperture simulations were performed using the code SimTrack developed at BNL [16]. In all cases the particles are tracked over $10^{6}$ turns with a momentum offset of $\delta p / p=1.24 \times 10^{-3}(3 \times$ the typical $\mathrm{rms}$ momentum spread, cf. Table II) and include all magnetic multipole field errors. The dynamic aperture is taken as the largest amplitude at which particles still survive the full $10^{6}$ turns, where 10 ratios of horizontal and vertical amplitudes are simulated.

Figure 8 shows a tune scan without beam-beam interactions for the Blue ATS lattice and the 2012 lattices. The separation between the horizontal and vertical tunes is kept

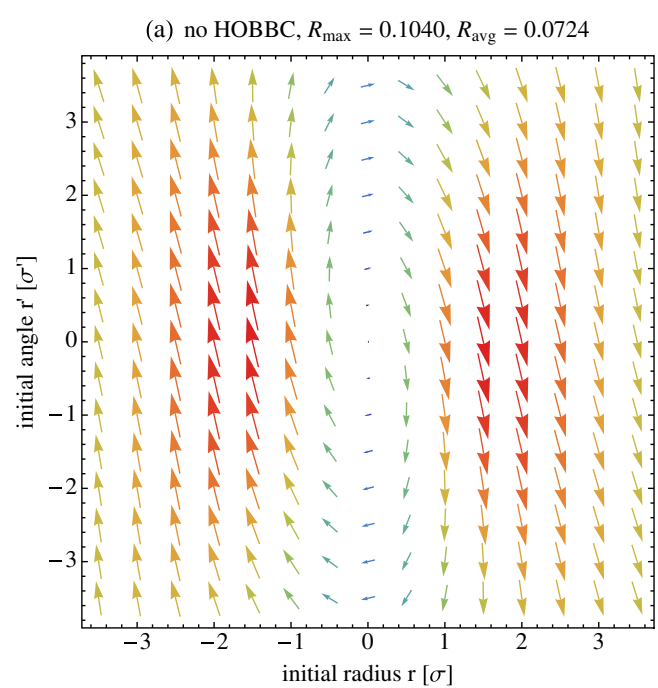

(b) HOBBC, $\Delta \psi=0 \mathrm{deg}, R_{\max }=0.0511, R_{\text {avg }}=0.0162$

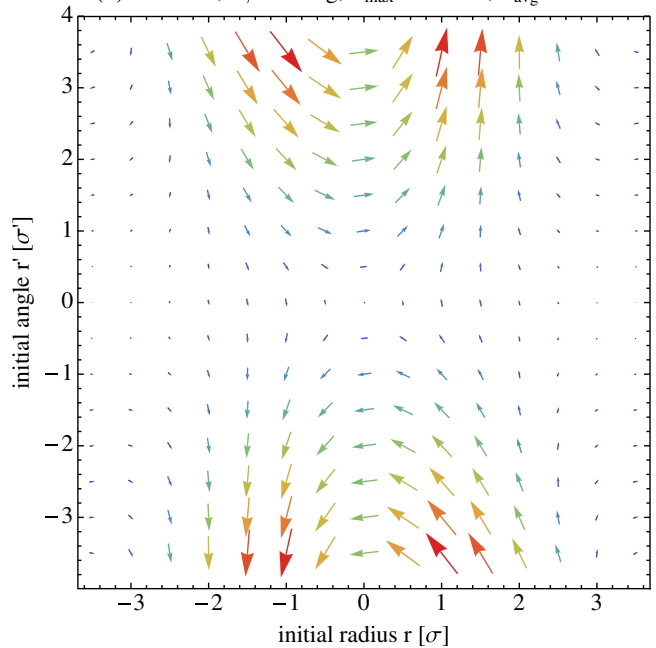

(c) HOBBC, $\Delta \psi=+5 \mathrm{deg}, R_{\max }=0.0606, R_{\mathrm{avg}}=0.0190$

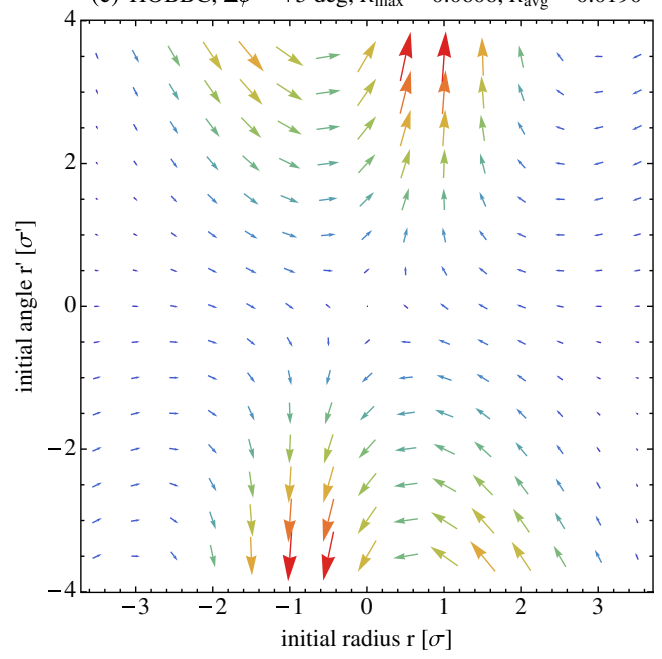

FIG. 7. Phase space vector $\vec{R}$ at IP10 as a function of the initial coordinates $\left(r_{i}, r_{i}^{\prime}\right)$ at IP8 without head-on beam-beam compensation in part (a), and deviation from full compensation in part (b) and (c). Part (b) shows the case of a matched electron beam size and current, part (c) has an additional linear phase error of +5 deg between the $\mathrm{p}+\mathrm{p}$ and $\mathrm{p}+\mathrm{e}$ interactions. 


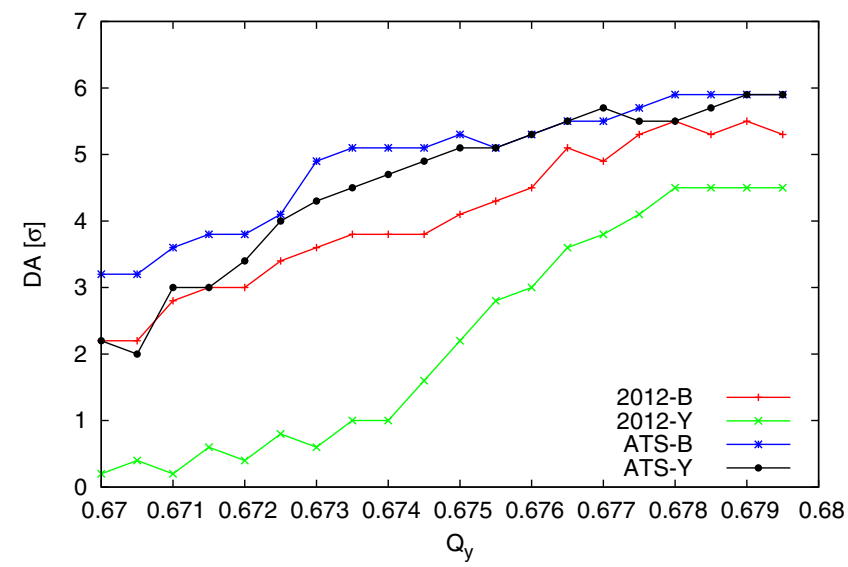

FIG. 8. Simulated dynamic aperture as a function of the vertical tune $Q_{y}$ without beam-beam interactions. B denotes the Blue, and $\mathrm{Y}$ the Yellow ring.

constant with $Q_{y}=Q_{x}-0.01$. A tune separation of 0.005 to 0.01 is typical for operation, and ensures that the tunes are close to the diagonal in the resonance diagram where the resonance density is low. A simulation showed that the dynamic aperture is reduced when the tunes are moved far away from the diagonal.

Figure 9 shows the dynamic aperture as a function of bunch intensity with beam-beam interactions for the 2012 lattices with and without electron lenses and the ATS lattices with electron lenses. Without compensation the dynamic aperture remains constant up to a bunch intensity of approximately $2.0 \times 10^{11} \mathrm{p} /$ bunch and then decreases as the beam-beam interactions become stronger. The 2012 lattice featured a phase advance of almost $k \pi$ between IP8 and IP10 in the Blue ring. This is not the case for the Yellow ring for which a clear degradation of the dynamic aperture is observed at high bunch intensity.

The dynamic aperture appears to increase with the bunch intensity $N_{p}$ for the case of 2012 lattice with the electron
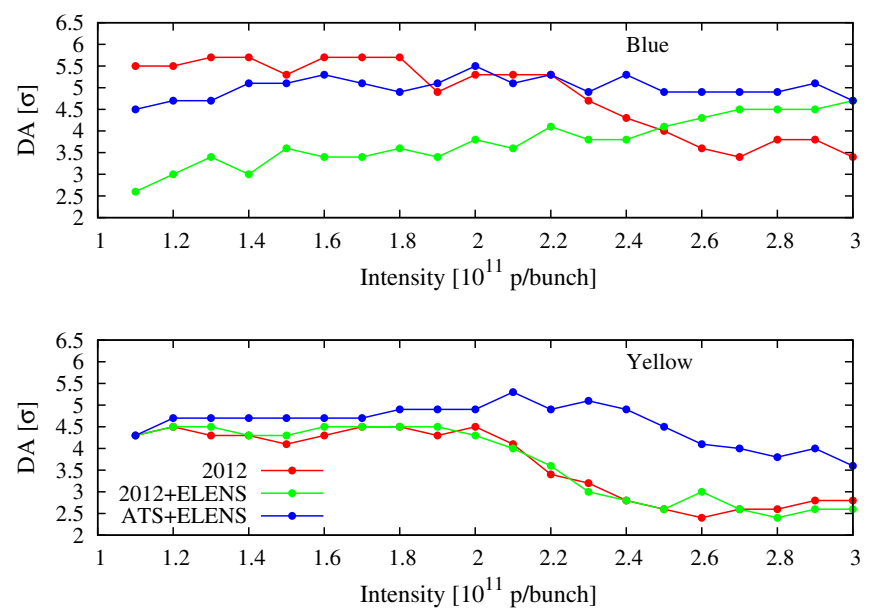

FIG. 9. Simulated dynamic aperture as a function of bunch intensity including beam-beam interactions. lens. This is, however, an artifact of the following phenomenon. To compare the dynamic apertures in simulations, the small amplitude lattice tunes were always set to $(0.68,0.67)$. In tune scan simulations these tunes were found to be favorable for operation with high bunch intensity (cf. Fig. 12 in Ref. [16] and Fig. 21 in Ref. [22]), and are consistent with the operational experience. In simulations the lattice tunes are adjusted upwards with increasing bunch intensities to compensate for the tune depression from the beam-beam interaction. The electron lenses only compensate for one of the two beam-beam interactions, and for the cases with electron lenses the lattice tunes are also adjusted upwards with increasing bunch intensity. With half head-on beam-beam compensation, and the small-amplitude tunes fixed at $(0.68,0.67)$, the reduced tune footprint is very close to the $2 / 3$ resonances resulting in a smaller dynamic aperture. The dynamic aperture can be increased by moving the tunes up and away from the $2 / 3$ resonances. A enhancement to the plot shown in Fig. 9 would require a tune scan at each of the bunch intensities sampled.

A proper matching of the phase advance to the electron lenses, as done for the 2015 ATS lattice, allows for a compensation of non-linear resonances. The dynamic aperture in this case becomes almost independent of the bunch intensity as observed for the ATS lattices.
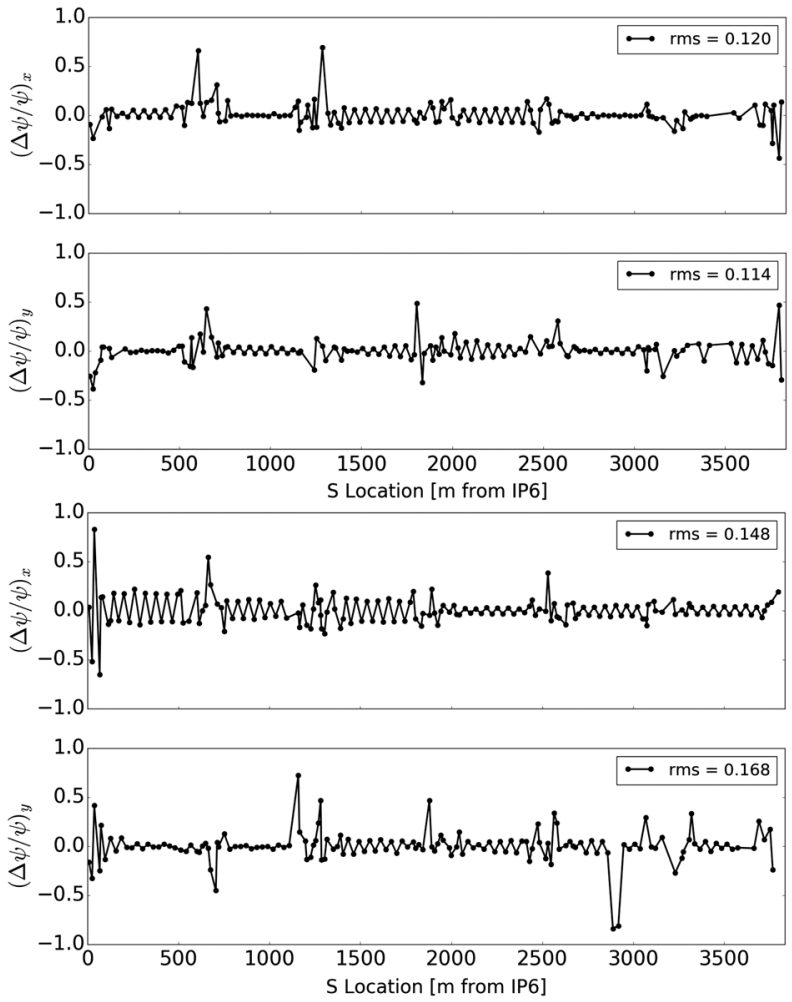

FIG. 10. Measured phase error $(\Delta \psi / \psi)_{x, y}$ for the Blue horizontal and vertical planes (top) and the Yellow horizontal and vertical planes (bottom). 
TABLE III. Design and measured lattice functions, phase advances and nonlinear chromaticities.

\begin{tabular}{|c|c|c|c|c|c|}
\hline \multirow[b]{2}{*}{ quantity } & \multirow[b]{2}{*}{ unit } & \multicolumn{2}{|c|}{ Blue } & \multicolumn{2}{|c|}{ Yellow } \\
\hline & & design & measured & design & measured \\
\hline lattice functions $\beta_{x} / \beta_{y}$ at IP6 & $\mathrm{m}$ & $0.85 / 0.85$ & $0.76 / 0.78$ & $0.85 / 0.85$ & $0.90 / 0.95$ \\
\hline lattice functions $\beta_{x} / \beta_{y}$ at IP8 & $\mathrm{m}$ & $0.85 / 0.85$ & $0.90 / 0.84$ & $0.85 / 0.85$ & $0.88 / 0.82$ \\
\hline lattice functions $\beta_{x} / \beta_{y}$ at e-lens & $\mathrm{m}$ & $15.0 / 15.0$ & $15.8 / 14.1$ & $15.0 / 15.0$ & $16.5 / 12.6$ \\
\hline phase advances $\Delta \psi_{x} / \Delta \psi_{y}$ IP8 to e-lens & $\operatorname{deg}$ & $180 / 180$ & $184 / 177$ & $180 / 180$ & $192 / 180$ \\
\hline rms beta-beat $(\Delta \beta / \beta)_{x} /(\Delta \beta / \beta)_{y}$ & $\%$ & $0 / 0$ & $11.7 / 12.1$ & $0 / 0$ & $11.7 / 14.1$ \\
\hline nonlinear chromaticity $\frac{1}{2} Q_{x}^{\prime \prime} / \frac{1}{2} Q_{y}^{\prime \prime}$ & .. & $-600 /-1350$ & $-500 /-1300$ & $-800 /-850$ & $-100 /-1100$ \\
\hline
\end{tabular}

\section{Lattice measurements}

The linear lattice functions were measured to verify the $\beta$-functions at the IPs and at the location of the electron lenses, as well as the phase advance between IP8 and the electron lenses. Figure 10 shows the measured $(\Delta \psi / \psi)_{x, y}$ for the Blue and Yellow rings. The measurement was taken by giving the proton beam a small kick and observing the resulting free betatron oscillations with beam position monitors (BPMs) around the ring. The $\Delta \psi_{x, y}$ shown in Fig. 10 is taken between neighboring BPMs, which in the arcs have a phase advance of close to $90^{\circ}$. In Table III the design and measured $\beta$-functions and phase advances $\Delta \psi_{x, y}$ are listed.
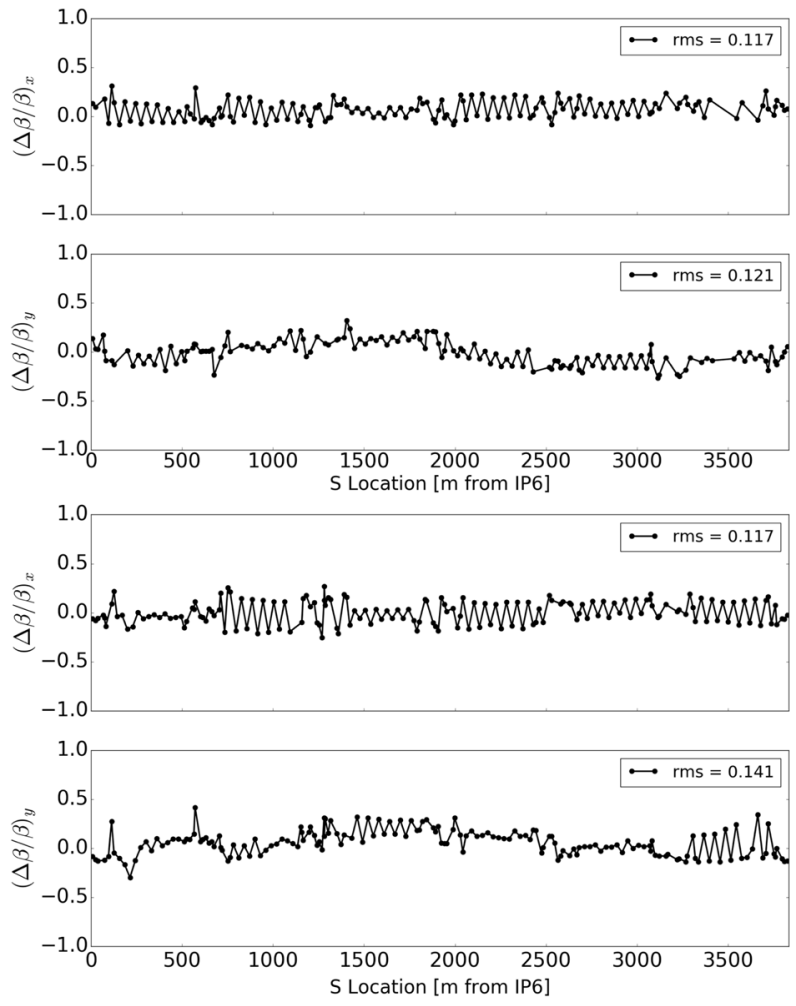

FIG. 11. Measured $\beta$-beat $(\Delta \beta / \beta)_{x, y}$ for the Blue horizontal and vertical planes (top) and the Yellow horizontal and vertical planes (bottom).
A large $\beta$-beat will reduce the effectiveness of the ATS lattice in compensating resonance driving terms. Measurements of the $\beta$-beat are shown in Fig. 11, and the rms values are also stated in Table III.

The nonlinear chromaticities $Q_{x, y}^{\prime \prime}$ were measured by observing the tune change with radius excursions of 0.5 , 1.0 and $1.25 \mathrm{~mm}$. The radius-weighted averages of the measured $Q_{x, y}^{\prime \prime}$ values are also listed in Table III. The standard errors of the measured $Q_{x, y}^{\prime \prime}$ values in the polynomial fits of the momentum-dependent tune functions range from 20 to 400, with an radius-averaged value of 110 . The agreement between design and measured $Q^{\prime \prime}$ is good in the Blue ring, and acceptable in the Yellow vertical plane. The discrepancy between the design and measured $Q^{\prime \prime}$ value in the Yellow vertical plane cannot be explained by the standard fit error of the measurement.

\section{EFFECT OF THE ELECTRON LENSES ON ORBIT AND TUNE}

Like the beam-beam interaction, an electron lens with a transverse offset has an effect on the orbit. The electron lens with and without an offset changes the coherent tune, and the tune spread. However, unless the electron lens drives an instability [cf. Eq. (6)] it is seen by the beam as a fixed lattice element and does not create coherent beam-beam oscillations modes, or change the coherent oscillation modes of colliding beams [31].

The effect of a RHIC electron lens on a hadron beam was first measured with gold beams (charge number $Z=79$, mass number $A=197$ ) in 2014. For example, the change in the vertical closed orbit slope $\Delta y_{c o}^{\prime}$ due to a vertical offset $y$ is the coherent beam-beam kick from the electron lens given by (cf. Ref. [18-20])

$\Delta y_{c o}^{\prime}=\frac{2 r_{p} Z}{\gamma_{A u} y A}\left(I_{e} L_{e}\right) \frac{1+\beta_{e}}{e \beta_{e} c}\left(1-\exp \left[\frac{-y^{2}}{2\left(\sigma_{e}^{2}+\sigma_{A u}^{2}\right)}\right]\right)$,

where $\gamma_{A u}$ is the Lorentz factor, and the gold beam is assumed to be ultrarelativistic with $\beta_{A u} \approx 1$. One arrives at Eq. (10) from the formula for the incoherent beam-beam 

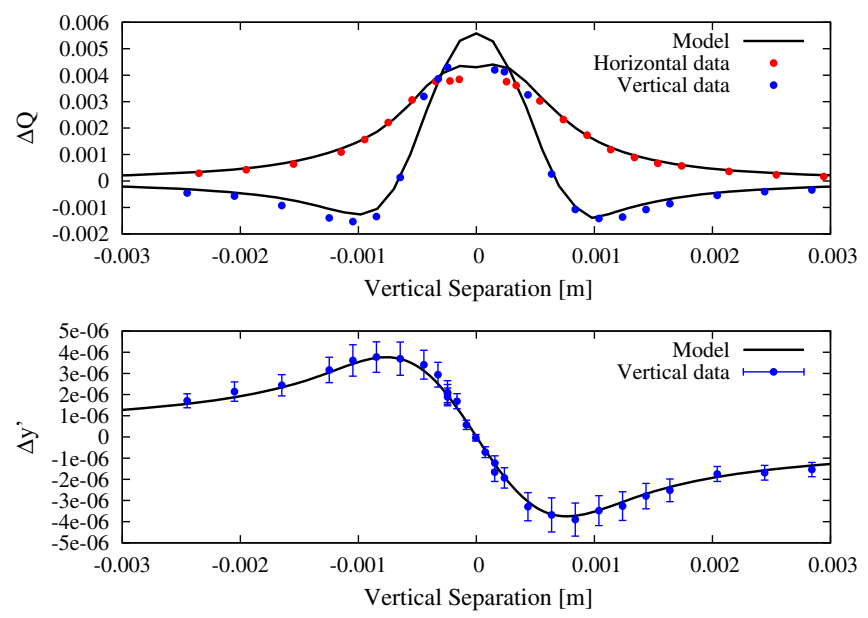

FIG. 12. Change of gold beam tunes $Q_{x, y}$ (top) and vertical closed orbit angle at the electron lens (bottom) in response to a vertical displacement.

kick from a beam of rms size $\sigma$ by (i) replacing $\sigma^{2}$ with $\left(\sigma_{e}^{2}+\sigma_{A u}^{2}\right) / 2$, and (ii) by adding a factor $1 / 2$ in the argument of the exponential function. The coherent beam-beam kick leads to closed orbit changes in the separation plane, and tune changes in both planes.

Figure 12 shows the measured change in the tunes $\Delta Q_{x, y}$ and the reconstructed orbit slope $\Delta y_{c o}^{\prime}$ at the location of the electron lens as a function of vertical separation. The measurement was taken with stochastically cooled gold beams [37-39], and the gold beam size at the lens changed slightly during the measurement due to the cooling $\left(\sigma_{\mathrm{Au}}=0.32-0.27 \mathrm{~mm}\right)$. The electron beam size was larger with $\sigma_{\mathrm{Au}}<\sigma_{e}=0.50 \mathrm{~mm}$. The measured tune values in Fig. 12 follow closely the expected values [32]. The lower part of Fig. 12 is a measurement of the coherent amplitudedependent beam-beam kick from the electron lens. Note that the small amplitude coherent beam-beam kick is a factor 2 smaller than the incoherent beam-beam kick shown in Fig. 1 [18-20]. The coherent beam-beam kick was previously measured with colliding $250 \mathrm{GeV}$ proton beams in RHIC [40]. The effect of the electron lens on the tune requires lattice tune changes when the current is ramped down in operation (as shown in Fig. 3, bottom).

\section{EFFECT OF THE ELECTRON LENSES ON TUNE SPREAD}

The primary function of the electron lens is the compression of the beam-beam generated tune footprint. To measure the width of the transverse tune distributions, beam transfer functions (BTFs) were used. These measure the complex beam response $R(Q)$ of a small harmonic dipole oscillation of variable frequency $2 \pi Q$. A nonzero particle distribution at tune $Q$ is given if $\operatorname{Im}(R)>0$, where $\operatorname{Im}(R)$ is the imaginary part of the transverse beam transfer function $[18,41,42]$.
With the operational tunes in $\mathrm{p}+\mathrm{p}$ operation (Table II) coherent beam-beam modes are excited in a BTF measurement, because $\left|Q_{\text {Blue }}-Q_{\text {Yellow }}\right| \lesssim\left|k_{c} \xi_{p}\right|$ in both transverse planes, where $k_{c}$ is the number of head-on beam-beam collisions per turn. Close tunes in the two rings allow for coupling of the beams and the existence of coherent modes, while separated tunes suppress the coherent modes [43], as is the case in $\mathrm{p}+\mathrm{Al}$ collisions in RHIC (see below).

The dominant coherent modes $Q_{\sigma}$ and $Q_{\pi}$ are the inphase and out-of-phase transverse oscillations of the two beams at the IP. $Q_{\sigma}$ is also the lattice tune and is at the edge of the incoherent tune distribution, $Q_{\pi}$ is outside of this distribution when the incoherent tune spread is only generated by the beam-beam interaction, and the $Q_{\pi}$ oscillation mode may therefore be unstable [43]. For round proton beams of equal size at the IP we expect for the dominated modes $Q_{\pi}-Q_{\sigma}=Y k_{c} \xi_{p}$, with the Yokoya factor $Y \approx 1.21$ [44].

Although it was possible to isolate the incoherent tune distribution in the presence of coherent modes from BTF measurements in simulations [41], this was not successful with measured BTFs. Figure 13 shows the reconstructed distribution widths $\operatorname{Im}(R) /|R|$ as a function of the excitation tune $Q$ for several cases. The distribution widens with the electron lens, and with 1 and 2 beam-beam collisions. However, the width of the reconstructed tune distribution is unchanged with beam-beam collisions and the electron lens due to the coherent modes, which determine the width of $\operatorname{Im}(R) /|R|$. The coherent $\sigma$ and $\pi$ modes are essentially unchanged after the electron lenses are added [31].

In a BTF measurement of a noncolliding beam interacting with the electron lens this is not a problem since no coherent modes are excited. To obtain the incoherent tune distribution widths with colliding beams BTF

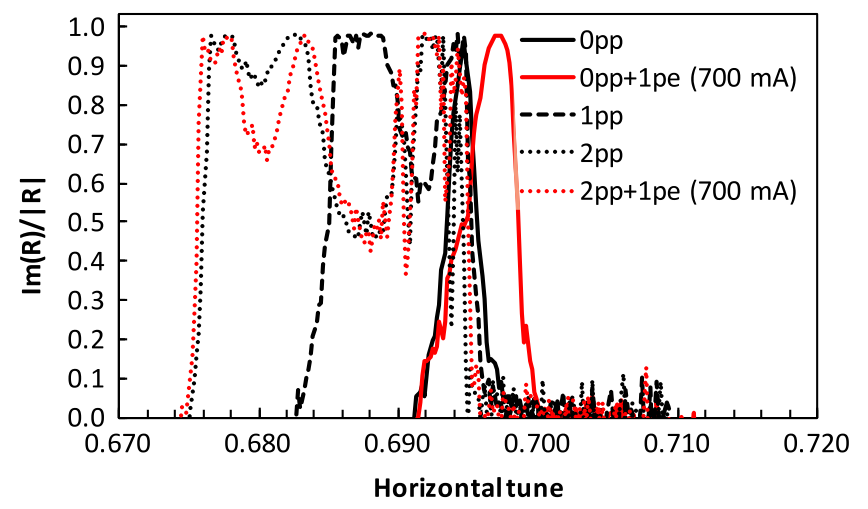

FIG. 13. Reconstructed tune distribution widths from BTF measurements without beam-beam interaction (solid black), with electron lens (solid red), with 1 (dashed black) and 2 (dotted black) beam-beam interactions, and with 2 beam-beam interactions and an electron lens (dotted red). The widths is of colliding beam distributions is determined by the distance of the coherent modes, not by the incoherent tune distributions. 
measurements were done in $\mathrm{p}+\mathrm{Al}$ collisions. Aluminum beams have fractional tunes near 0.225 far away from the $\mathrm{p}$ beam tunes near 0.69 (Table II). The separation of Blue and Yellow ring tunes is large enough to suppress the excitation of coherent beam-beam modes, and the incoherent distribution width can be reconstructed. The fractional tunes for ions were chosen for small beam losses during transition crossing, and the fractional tunes for protons were chosen to accommodate a large beam-beam generated tune spread while preserving the polarization. All ions other than protons cross the transition energy in RHIC, and ion beams have beam-beam parameters typically a factor $2-3 \times$ smaller than proton beams.

A number of scans were made to measure the parametric dependence of the incoherent tune distribution width as a function of the electron lens parameters. Figure 14 shows the incoherent tune distribution width as a function of the electron beam current $I_{e}$ for a constant electron beam size of $\sigma_{e}=0.55 \mathrm{~mm}$. Figure 15 shows the tune distributions as a function of the electron beam size $\sigma_{e}$ with an electron beam current $I_{e}=900 \mathrm{~mA}$. In these scans the rms proton beam size in the electron lens, as measured by the ionization profile monitor (IPM), was $\sigma_{p}=0.60 \mathrm{~mm}$ and within the measurement error constant throughout the measurement.

The beam-beam parameters generated by the proton beam and electron lens $\xi_{p, e}$ are given by Eq. (4), and the tune shift of a particle at amplitude $a$ from the center of either a proton or electron beam is [20]

$$
\delta Q_{p, e}(a)=\xi_{p, e} \frac{2}{\alpha}\left[1-\exp \left(-\frac{\alpha}{2}\right) I_{0}\left(\frac{\alpha}{2}\right)\right]
$$

with $\alpha=\frac{1}{2}\left(a / \sigma_{p, e}\right)^{2} . I_{0}$ is the modified Bessel function of order zero. The beam-beam generated tune spread over the amplitude range from zero to $a$ is then

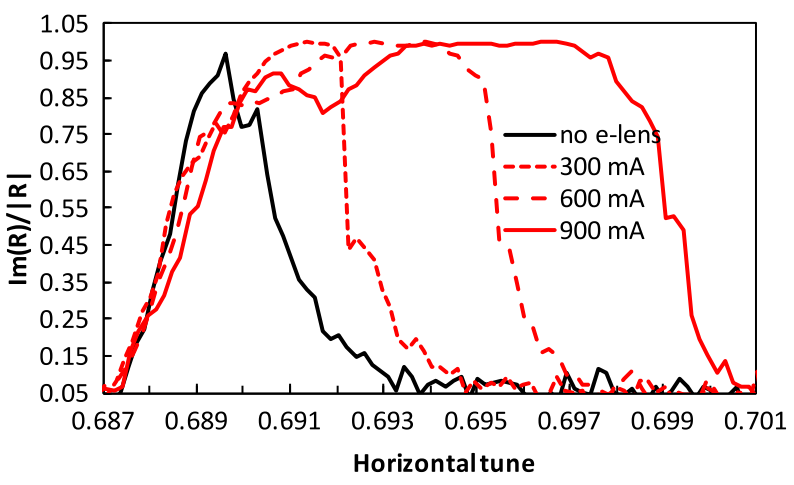

FIG. 14. Measured $\mathrm{p}$ beam tune distribution width as a function of the electron beam current without beam-beam collisions and with an electron beam size of $\sigma_{e}=0.55 \mathrm{~mm}$. The proton beam size is $\sigma_{p}=0.60 \mathrm{~mm}$. The curves are aligned to the left with an offset for better visibility of the effect [1].

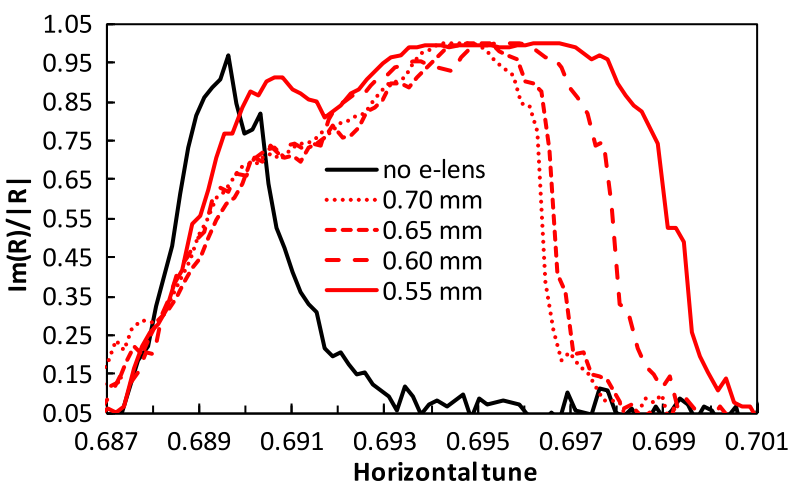

FIG. 15. Measured $\mathrm{p}$ beam tune distribution width as a function of the electron beam size $\sigma_{e}$ without beam-beam collisions and with an electron current of $900 \mathrm{~mA}$. The proton beam size is $\sigma_{p}=0.60 \mathrm{~mm}$. The curves are aligned to the left with an offset for better visibility of the effect.

$$
\Delta Q_{b b}(a)=\left|\xi_{p, e}-\delta Q_{p, e}(a)\right|,
$$

and it is assumed that the total tune spread $\Delta Q_{\text {tot }}$ is given by

$$
\Delta Q_{\mathrm{tot}}^{2}=\Delta Q_{0}^{2}+\Delta Q_{b b}^{2}(a)
$$

where $\Delta Q_{0}$ is the tune spread without beam-beam interactions. The incoherent tune spread $Q_{0}$ has two principal sources: (i) chromaticity in conjunction with the momentum distribution, and (ii) nonlinear magnetic fields including field errors in the triplets. Neither of these sources are correlated with the beam-beam interaction, justifying the use of Eq. (13). With a typical $Q^{\prime} \approx+2$ and the momentum spreads in Table II, $\Delta Q_{0} \approx 0.003$ from chromaticity for $3 \sigma_{\delta}$ although it can be significantly larger in an experimental setup. The tune spread $Q_{0}$ from nonlinear magnetic fields is best determined by simulations and is typically a few 0.001 for $3 \sigma_{p}$ depending on the $\beta^{*}$ values in all IPs [22].

Figure 16 shows a comparison of the measured increase in the tune distribution width due to the electron lens for the current scan shown in Fig. 14 and the beam size scan shown in Fig. 15 in parts (a) and (b) respectively. The measured width of the tune distribution is taken at $|\operatorname{Im}(R) /(R)|=0.1$. The measurement matches the calculation for a fitted value $a=2.5 \sigma_{p}$. There are only few particles at amplitudes $a>2.5 \sigma_{p}$ and the BTF signal $R$ is weak with small particle numbers.

Finally, Fig. 17 shows the tune distribution width without beam-beam interaction, with beam-beam interaction, and with an increasing electron beam current, with an electron beam size of $\sigma_{e}=0.65 \mathrm{~mm}\left(\sigma_{p}=0.60 \mathrm{~mm}\right.$ over the course of the measurement). The measurement is taken with a proton beam that was colliding with an aluminum beam. The tune spread increases with the beam-beam interaction and is gradually compressed with an increasing electron beam current $I_{e}$ up to the initial tune distribution with $I_{e}=750 \mathrm{~mA}$. Note that a further increase in $I_{e}$ does 

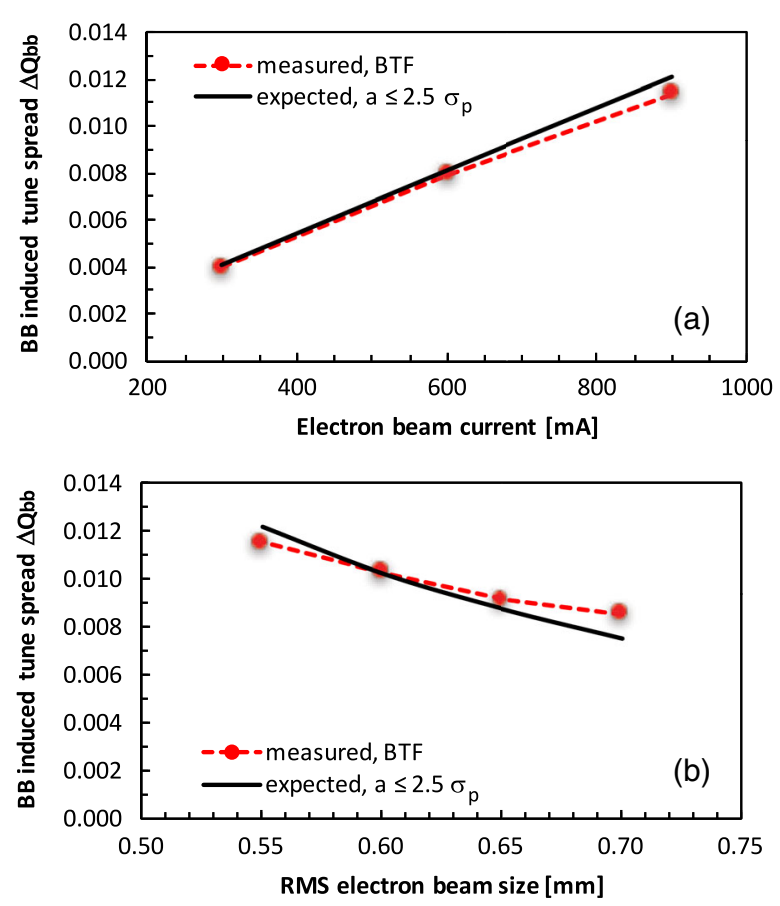

FIG. 16. Comparison of measured and calculated increase in the tune spread of the electron lens in an electron beam current scan (a) and electron beam size scan (b). The measured width of the tune distribution is taken at $|\operatorname{Im}(R) /(R)|=0.1$, the calculation is for an amplitude range $a$ from zero to $2.5 \sigma_{p}$.

not lead to a further reduction in the tune spread. The initial tune spread is primarily due to nonzero chromaticity and momentum spread, and cannot be compensated for with the electron lens. For further increasing currents one expects the tune distribution width to widen again.

Based on the data in Fig. 17, Fig. 18 shows a comparison between measured and expected total tune spread $\Delta Q_{\text {tot }}$ as a function of the electron beam current $I_{e}$. The expected total tune spread is based on the BTF measured tune spread $\Delta Q_{0}$ and the beam-beam generated tune spread $\Delta Q_{b b}(a)$

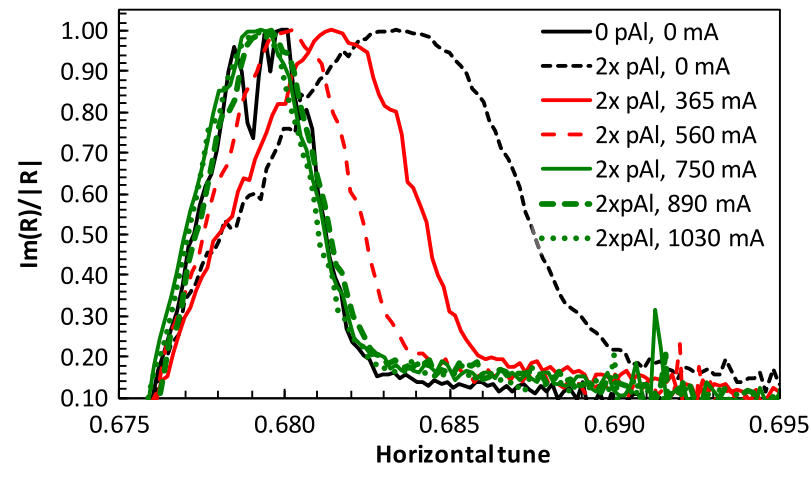

FIG. 17. Tune distribution width reduction with the electron lens, measured in the proton beam with $\mathrm{p}+\mathrm{Al}$ collisions. The distribution widens due to two beam-beam interactions, and narrows again with the electron lens. The curves are aligned to the left with an offset for better visibility of the effect.

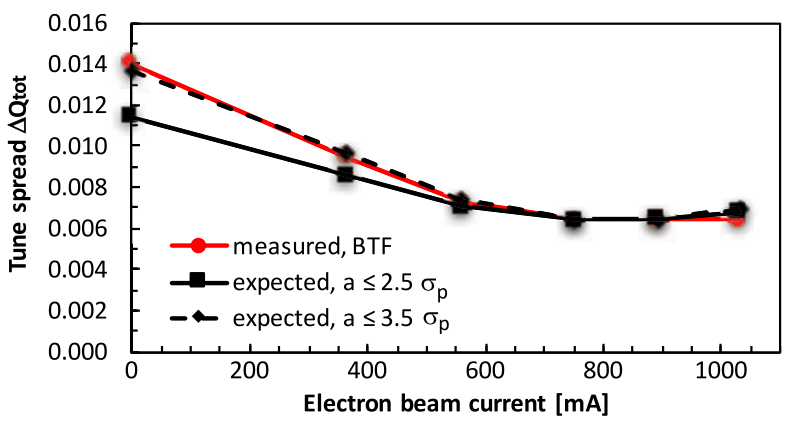

FIG. 18. Total tune spread $\Delta Q_{\mathrm{tot}}$ as a function of the electron beam current $I_{e}$. The measured total tune spread is based on BTF measurements. The expected total tune spread is based on the measured tune spread $Q_{0}$ and the beam-beam parameters $\xi_{\mathrm{Al}}$ and $\xi_{e}$ using Eq. (13), and shown for $a \leq 2.5 \sigma_{p}$ and $a \leq 3.5 \sigma_{p}$.

using Eqs. (12) and (13), where $\Delta Q_{b b}(\infty)=\left|\left(2 \xi_{A l}+\xi_{e}\right)\right|$. The beam-beam parameter $\xi_{\mathrm{Al}}$ is obtained by a measurement of the bunch intensity $N_{\mathrm{Al}}$ with a direct currentcurrent transformer (DCCT) and the scaled emittance $\epsilon_{n}$ with an ionization profile monitor (IPM). During $\mathrm{p}+\mathrm{Al}$ operation the IPM reported emittance was on average 7\% smaller than the luminosity derived emittance. Beam-beam parameters quoted here, and in Ref. [1], use the emittance consistent with the independent luminosity calibration, and IPM emittances are scaled. Note that emittance measurements have typical errors of $\geq 10 \%$. $\xi_{\mathrm{Al}}$ changes from -0.0080 to -0.0068 over the course of the measurement. The beam-beam parameter $\xi_{e}$ is obtained from the electron beam current $I_{e}$ and the electron beam size [Eq. (4)], and $\xi_{e}$ reaches +0.0174 at $1030 \mathrm{~mA}$. Figure 18 shows the expected tune spread $\Delta Q_{\text {tot }}$ for $a \leq 2.5 \sigma_{p}$, the value that provided a good fit in Fig. 16(a) and (b), and for $a \leq 3.5 \sigma_{p}$, which is a better fit in this case.

\section{BEAM LOSS RATE, EMITTANCE GROWTH, AVERAGE LUMINOSITY, AND EXPERIMENTAL BACKGROUND WITH ELECTRON LENSES}

The electron lenses can introduce additional beam loss and emittance growth and thereby reduce the average store luminosity. They can also increase the experimental background rates. However, the lenses are only needed as long as the lattice alone cannot support the beam-beam parameter $\xi_{p}$, and were gradually turned off after a total on-time of 0.5 to $1.5 \mathrm{~h}$ for the last 50 stores of the run with the highest luminosities.

Time-dependent variations in the electron beam current, position or size can create additional beam loss and emittance growth, and even a lens with perfectly stable current, position and size can have an effect on the longterm stability of the chaotic particle motion of the circulating protons. The lens represents an additional strong nonlinearity, as strong as another beam-beam interaction, 
and its placement in the lattice, and the size and shape of the electron beam affect the proton motion. These effects were studied in simulations [16] and guided the specifications for the electron lenses [14] together with the experience from the Tevatron lenses [4-7,9]. The most important specifications for the operation were a current ripple of $\delta I_{e} / I_{e} \leq 0.1 \%$, a Gaussian electron beam shape, and an electron beam size equal or larger than the proton beam size, $\sigma_{e} \geq \sigma_{p}[14,16]$.

In order to evaluate the effect of the electron lenses on the beam loss rate, emittance growth, and experimental background, 30 stores were selected up to the time when the longitudinal polarization direction in one of the experiments was changed, which could have affected the store conditions. In these 30 stores, the Yellow electron lens was left on longer in two stores, for $6.75 \mathrm{~h}$ and $5.28 \mathrm{~h}$ respectively. In all other stores the Yellow electron lens was on between $0.45 \mathrm{~h}$ and $1.83 \mathrm{~h}$.

In polarized proton operation both the beam intensity and luminosity can usually be well fitted to a double exponential decay. For the intensity we have

$$
N_{p}(t)=N_{p 1} \exp \left(-t / \tau_{1}\right)+N_{p 2} \exp \left(-t / \tau_{2}\right) .
$$

Subscript 1 denotes the fast decaying component, with $N_{p 1}$ typically $10 \%$ of $N_{p}(0)$ and $\tau_{1} \approx 0.7 \mathrm{~h}$, and $\tau_{2}$ the slow decaying component with $\tau_{2}$ of order $100 \mathrm{~h}$. Figure 19 shows the slow component beam loss rate $1 / \tau_{2}$ for the 30 selected stores. The two stores with a long electron lens on-time are marked as red open circles. The beam loss rates are $0.5-1.0 \% / \mathrm{h}$ higher than the average of the 30 stores, and $1-2 \% / \mathrm{h}$ higher than the stores with the lowest loss rates.

Figure 20 shows the Yellow horizontal and vertical emittances, as measured by the ionization profile monitor (IPM) for the same 30 stores, and the stores with the Yellow electron lens on for 6.75 and $5.28 \mathrm{~h}$ respectively are shown in red again. The average horizontal and vertical rms emittance growth for the 30 stores is $0.63 \mu \mathrm{m}(+27 \%)$ and $0.63 \mu \mathrm{m}(+27 \%)$ compared to $0.82 \mu \mathrm{m}(+34 \%)$ and $0.79 \mu \mathrm{m}(+33 \%)$ for the average of two stores with the long

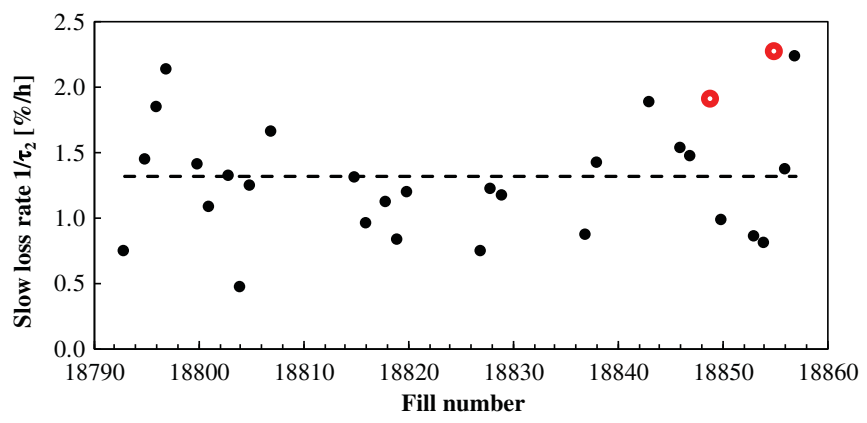

FIG. 19. Yellow beam loss rate of 30 stores. The two red marked stores had the Yellow electron lens on for $6.75 \mathrm{~h}$ and $5.28 \mathrm{~h}$ respectively, for all other stores the lenses were on between 0.45 to $1.83 \mathrm{~h}$.
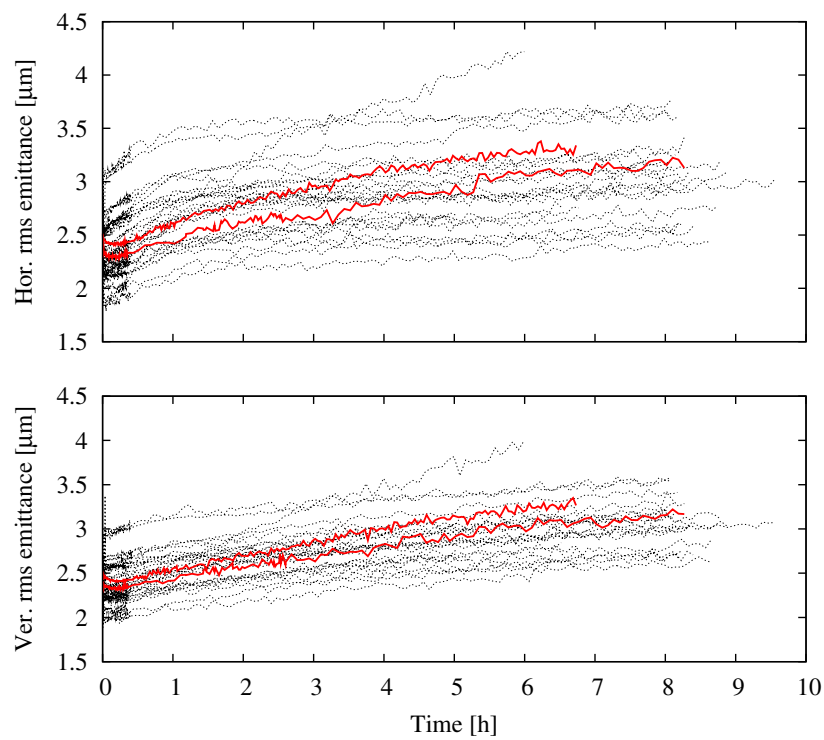

FIG. 20. Horizontal (top) and vertical (bottom) rms emittance of 30 stores. The two red marked stores had the Yellow electron lens on for $6.75 \mathrm{~h}$ and $5.28 \mathrm{~h}$ respectively, for all other stores the lenses were on between 0.45 to $1.83 \mathrm{~h}$.

electron lens on-time. Thus, if the lenses were on for a full store the additional emittance increase at the end of the store is about $6 \%$, or about $3 \%$ on average during the store. Since the luminosity decays during the store, the corresponding reduction in the integrated luminosity is expected to be less than $3 \%$ [Eq. (1)] for a well-tuned machine and electron lens.

A rough estimate for the characteristic emittance growth time for white noise current fluctuations can be calculated as [9]

$$
\tau_{e}^{\epsilon}=\frac{1}{4 \pi^{2} f_{0} \xi_{e}^{2}\left(\delta I_{e} / I_{e}\right)^{2}}
$$

where $f_{0}$ is the revolution frequency. Based on the Tevatron electron lens experience, the current ripple had a specification of $\delta I_{e} / I_{e} \leq 0.1 \%$, which was confirmed on the electron lens test bench via a voltage ripple measurement [11]. With $f_{0}=78 \mathrm{kHz}$ and $\xi_{e}=+0.01 \mathrm{Eq}$. (15) yields a lower bound of $\tau_{e}^{\epsilon} \approx 1 \mathrm{~h}$. The measured emittance growth time is about 25 h (Fig. 20).

Since the electron lenses were on for a variable time, a large impact on the instantaneous luminosity should be visible in the average store luminosity as long as the lenses cover the time when the lattice alone cannot support the beam-beam parameter $\xi_{p}$. Figure 21 shows the average store luminosity for the last 40 stores of the 2015 run with a store length of $6 \mathrm{~h}$ or longer, when the machine was at peak performance. There is no correlation between the time the lenses were on and the average luminosity.

While there is an effect of the electron lenses on the beam loss rates $(0.5-1.0 \% / \mathrm{h})$ and emittance growth rate $(+3 \%$ 


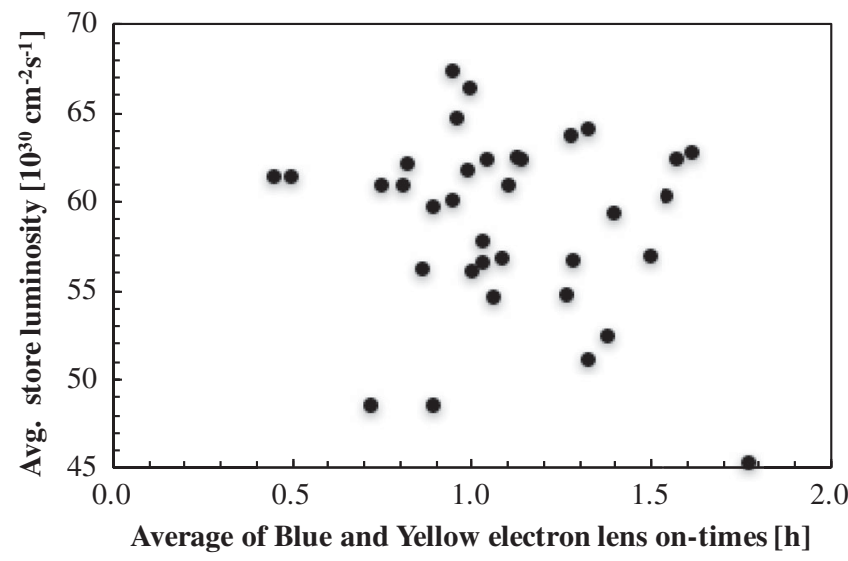

FIG. 21. Average store luminosity $\mathcal{L}_{\text {avg }}$ as a function of the Blue and Yellow average electron lens on-time for the last 40 stores of the 2015 run with a store length of $6 \mathrm{~h}$ or longer.

store average), this is not detectable in the average store luminosity $\mathcal{L}_{\text {avg }}$ for the parameters of the 2015 run.

The experimental background at PHENIX, one of the two experiments, is assessed through two sets of background counters, installed on both sides and close to the PHENIX experiment, and positioned such that they are sensitive to beam loss in the final focus triplet magnets. These quadrupoles are at the locations with the largest $\beta$-functions in RHIC. In 2015 the $\beta$-functions at the two experiments STAR and PHENIX were the same, and the signals of the counters at PHENIX are indicative of the machine-generated background conditions for both experiments.

Figure 22 shows the location of two of these scintillator detectors, N2 and N3, on the north side of the detector, which are sensitive to the background generated by the

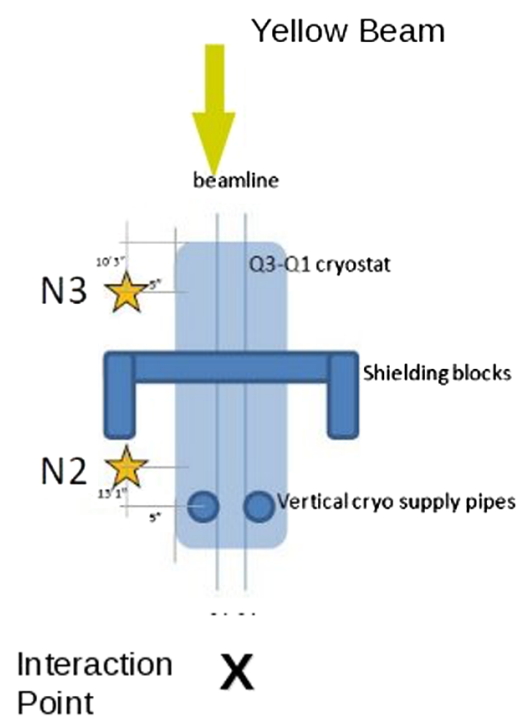

FIG. 22. Location of two experimental background monitors (scintillators), $\mathrm{N} 2$ and $\mathrm{N} 3$, in the PHENIX interaction region.

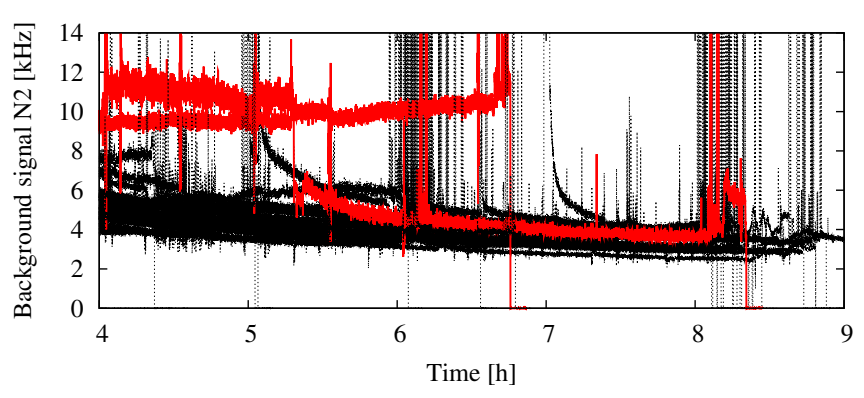

FIG. 23. PHENIX N2 background signal. The two red marked stores had the Yellow electron lens on for $6.75 \mathrm{~h}$ and $5.28 \mathrm{~h}$ respectively, all other stores had the lenses on between 0.45 to $1.83 \mathrm{~h}$. Zero (the beginning of the store) is suppressed on the horizontal axis to better show the change in the background signal when the lenses are turned off.

Yellow beam. Figure 23 shows the N2 signal, which is a factor 2-3 times higher for the two stores with the Yellow electron lens on for 6.75 and $5.28 \mathrm{~h}$ than for the comparison stores with shorter on-time. This higher background rate was acceptable, and background rates in 2015 were still a factor 3-4 times smaller than in 2012 even with doubled luminosity.

\section{SUMMARY}

A head-on beam-beam compensation scheme has been implemented in RHIC consisting of electron lenses that reduce the beam-beam generated tune spread and a lattice that minimizes the beam-beam generated resonance driving terms. The lattice requires a phase advance of $k \pi, k$ being an integer, between the head-on beam-beam interactions to be compensated and the electron lenses. The transversely Gaussian electron beam of the lens needs to be matched to the hadron beam in size and current for compensation.

The lattice properties were measured, and $\beta$-functions and phase advances are close to the design values. The effect of the electron lens on the hadron beams was measured (orbit, tune and tune spread) and found to be in agreement with expectations. The incoherent tune spread generated by the electron lenses was measured and is in agreement with BTF based measurements of the tune distribution width assuming that particles in the amplitude range from zero to $2.5 \sigma_{p}$ are detected in the measurement. The beam-beam generated tune spread is indeed compensated by the electron lens, and in this case the measured tune distribution width is in agreement with expectations assuming particles in the amplitude range from zero to $3.5 \sigma_{p}$ are detected.

For a well-tuned machine the electron lenses increased the beam loss rate by $0.5-1.0 \% / \mathrm{h}$, the emittance by an additional $6 \%$ over an $8 \mathrm{~h}$ store, and the experimental background-as measured by scintillators near the detector - by a factor 2-3. Only the increased beam loss rate is large enough to be of concern, and resulted in 
operating the electron lenses only in the early part of the store when the beam-beam parameter $\left|\xi_{p}\right|$ is largest.

In 2015 the head-on beam-beam compensation scheme was used for the first time operationally with polarized protons at $100 \mathrm{GeV}$, leading to almost doubled peak and average luminosities. Of this gain, and based on the maximum measured beam-beam parameter $\left|\xi_{p}\right|$ with and without the electron lens, $67 \%$ can be attributed to the new lattice alone, and $91 \%$ to the lattice and electron lenses combined.

\section{ACKNOWLEDGMENTS}

The authors are thankful for technical support of the groups in the Collider-Accelerator Department and Superconducting Magnet Division at Brookhaven National Laboratory. We are also grateful for discussions and support to V. Shiltsev, A. Valishev, T. Sen, and G. Stancari, FNAL, who generously shared the Tevatron experience with us; N. Milas, ESS; X. Buffat, R. DeMaria, W. Herr, J.-P. Koutchouk, T. Pieloni, F. Schmidt, and F. Zimmerman, CERN; U. Dorda, DESY; K. Ohmi, KEK; J. Qiang, LBNL; V. Kamerdziev, FZ Jülich; A. Kabel, formerly SLAC and P. Görgen, formerly TU Darmstadt. We are thankful to the US LHC Accelerator Research Program (LARP) for support of beam-beam simulations. This work was supported by Brookhaven Science Associates, LLC, under Contract No. DE-AC0298CH10886 with the U.S. Department of Energy.

[1] W. Fischer, X. Gu, Z. Altinbas, M. Costanzo, J. Hock, C. Liu, Y. Luo, A. Marusic, R. Michnoff, T. A. Miller, A. I. Pikin, V. Schoefer, P. Thieberger, and S. M. White, Operational Head-On Beam-Beam Compensation in the Relativistic Heavy Ion Collider, Phys. Rev. Lett. 115, 264801 (2015).

[2] G. Arzelia et al., The Orsay compensated colliding beam rings (D.C.I.), Proceedings of the 8th International Conference on High Energy Accelerators (CERN, Geneva, 1971), pp. 150.

[3] M. Bergher et al., Status report on D.C.I., IEEE Trans Nucl. Sci. NS-26, 3559 (1979).

[4] V. Shiltsev and A. Zinchenko, Electron beam distortions in beam-beam compensation setup, Phys. Rev. ST Accel. Beams 1, 064001 (1998).

[5] V. Shiltsev, Y. Alexahin, K. Bishofberger, V. Kamerdzhiev, G. Kuznetsov, and X.-L. Zhang, Experimental Demonstration of Colliding-Beam-Lifetime Improvement by Electron Lenses, Phys. Rev. Lett. 99, 244801 (2007).

[6] X.-L. Zhang, K. Bishofberger, V. Kamerdzhiev, V. Lebedev, V. Shiltsev, R. Thurman-Keup, and A. Tollestrup, Generation and diagnostics of uncaptured beam in the Fermilab Tevatron and its control by electron lenses, Phys. Rev. ST Accel. Beams 11, 051002 (2008).

[7] V. Shiltsev, K. Bishofberger, V. Kamerdzhiev, S. Kozub, M. Kufer, G. Kuznetsov, A. Martinez, M. Olson, H. Pfeffer,
G. Saewert, V. Scarpine, A. Seryi, N. Solyak, V. Sytnik, M. Tiunov, L. Tkachenko, D. Wildman, D. Wolff, and X.-L. Zhang, Tevatron electron lenses: Design and operation, Phys. Rev. ST Accel. Beams 11, 103501 (2008).

[8] G. Stancari, A. Valishev, G. Annala, G. Kuznetsov, V. Shiltsev, D. A. Still, and L. G. Vorobiev, Collimation with Hollow Electron Beams, Phys. Rev. Lett. 107, 084802 (2011).

[9] V. Shiltsev, Electron Lenses for Super-Colliders (Springer, New York, 2016).

[10] X. Gu, M. Okamura, A. Pikin, W. Fischer, and Y. Luo, The effects of realistic pancake solenoids on particle transport, Nucl. Instrum. Methods Phys. Res., Sect. A 637, 190 (2011).

[11] X. Gu, M. Okamura, A. Pikin, W. Fischer, and Y. Luo, The electron lens test bench for the relativistic heavy ion collider at Brookhaven National Laboratory, Nucl. Instrum. Methods Phys. Res., Sect. A 743, 56 (2014).

[12] X. Gu, Z. Altinbas, M. Costanzo, W. Fischer, D. M. Gassner, J. Hock, Y. Luo, T. Miller, Y. Tan, P. Thieberger, C. Montag, and A. I. Pikin, Transverse profile of the electron beam for the RHIC electron lenses, Nucl. Instrum. Methods Phys. Res., Sect. A 798, 36 (2015).

[13] P. Thieberger, Z. Altinbas, C. Carlson, C. Chasman, M. Costanzo, C. Degen, K. A. Drees, W. Fischer, D. Gassner, X. Gu, K. Hamdi, J. Hock, A. Marusic, T. Miller, M. Minty, C. Montag, Y. Luo, A. I. Pikin, and S. M. White, High energy Coulomb-scattered electrons for relativistic particle beam diagnostics, Phys. Rev. Accel. Beams 19, 041002 (2016).

[14] X. Gu et al., Electron lenses for head-on beam-beam compensation in RHIC, Phys. Rev. Accel. Beams 20, 023501 (2017).

[15] M. A. Furman and M. S. Zisman, Handbook of Accelerator Physics and Engineering, 2nd ed. (World Scientific, Singapore, 2013), p. 311.

[16] Y. Luo, W. Fischer, N. P. Abreu, X. Gu, A. Pikin, and G. Robert-Demolaize, Six-dimensional weak-strong simulation of head-on beam-beam compensation in the Relativistic Heavy Ion Collider, Phys. Rev. ST Accel. Beams 15, 051004 (2012).

[17] W. Fischer et al., CERN Report No. CERN-2014-004, 2014, p. 109.

[18] R. Talman, Multiparticle phenomena and Landau damping, AIP Conf. Proc. 153, 789 (1987).

[19] K. Hirata, Coherent betatron oscillation modes due to beam-beam interaction, Nucl. Instrum. Methods Phys. Res., Sect. A 269, 7 (1988).

[20] E. Keil, CERN Report No. CERN 95-06, 1995, p. 539.

[21] W. Fischer, Y. Luo, and C. Montag, Report No. BNL91114-2010-IR, C-AD/AP/359, 2010.

[22] Y. Luo, W. Fischer, and S. White, Analysis and modeling of proton beam loss and emittance growth in the Relativistic Heavy Ion Collider, Phys. Rev. Accel. Beams 19, 021001 (2016).

[23] V. Schoefer et al., in International Particle Accelerator Conference 2015, Richmond, VA, USA (JACoW, 2015), pp. 2384-2386.

[24] A. Luccio and M. Syphers, BNL Internal Report No. AGS/ RHIC/SN-068, 1997. 
[25] Y. K. Batygin and T. Katayama, Spin depolarization due to beam-beam collisions, Phys. Rev. E 58, 1019 (1998).

[26] A. Zelenski et al., Polarized $H^{-}$-jet polarimeter for absolute proton polarization measurements in RHIC, AIP Conf. Proc. 675, 954 (2002).

[27] T. Wise et al., in Proceedings SPIN 2004, Trieste, Italy (World Scientific, Singapore, 2004), p. 757.

[28] V. Schoefer et al., in Proceedings of the 3rd International Particle Accelerator Conference, New Orleans, LA, 2012 (IEEE, Piscataway, NJ, 2012), p. 184.

[29] http://www.rhichome.bnl.gov/RHIC/Runs/.

[30] A. Burov, V. Danilov, and V. Shiltsev, Transverse beam stability with an electron lens, Phys. Rev. E 59, 3605 (1999).

[31] S. White, M. Blaskiewicz, W. Fischer, and Y. Luo, CERN Report No. CERN-2014-004, 2014, p. 133.

[32] W. Fischer, X. Gu, S. M. White, Z. Altinbas, D. Bruno, M. Costanzo, J. Hock, A. Jain, Y. Luo, C. Mi, R. Michnoff, T. A. Miller, A. I. Pikin, T. Samms, Y. Tan, R. Than, and P. Thieberger, in Proceedings of the International Particle Accelerator Conference 2014, Dresden, Germany (JACoW, Geneva, 2013), p. 913.

[33] V. H. Ranjbar, F. Meot, M. Bai, D. T. Abell, and D. Meiser, Polarization response of RHIC electron lens lattices, Phys. Rev. Accel. Beams 19, 101002 (2016).

[34] S. Fartoukh, Achromatic telescopic squeezing scheme and application to the LHC and its luminosity upgrade, Phys. Rev. ST Accel. Beams 16, 111002 (2013).

[35] S. White, W. Fischer, and Y. Luo, Report No. BNL105550-2014-IR, C-A/AP/519, 2014.
[36] E. Cruz-Alaniz, D. Newton, R. Tomás, and M. Korostelev, Design of the large hadron electron collider interaction region, Phys. Rev. ST Accel. Beams 18, 111001 (2015).

[37] M. Blaskiewicz and J. M. Brennan, Bunched beam stochastic cooler in a collider, Phys. Rev. ST Accel. Beams 10, 061001 (2007).

[38] M. Blaskiewicz, J. M. Brennan, and F. Severino, Operational Stochastic Cooling in the Relativistic Heavy Ion Collider, Phys. Rev. Lett. 100, 174802 (2008).

[39] M. Blaskiewicz, J. M. Brennan, and K. Mernick, ThreeDimensional Stochastic Cooling in the Relativistic Heavy Ion Collider, Phys. Rev. Lett. 105, 094801 (2010).

[40] S. White, Ph.D. thesis, University Paris-Sud 11, LAL 10-154, 2010; Report No. CERN-THESIS-2010-139, 2010.

[41] P. Görgen, O. Boine-Frankenheim, and W. Fischer, Beam transfer functions for relativistic proton bunches with beam-beam interactions, Nucl. Instrum. Methods Phys. Res., Sect. A 777, 43 (2015).

[42] A. W. Chao, Physics of Collective Beam Instabilities in High Energy Accelerators (John Wiley \& Sons., New York, 1993), Ch. 5.

[43] Y. Alexahin, A study of the coherent beam-beam effect in the framework of the Vlasov perturbation theory, Nucl. Instrum. Methods Phys. Res., Sect. A 480, 253 (2002).

[44] K. Yokoya and H. Koiso, Tune shift of coherent beambeam oscillations, Part. Accel. 27, 181 (1990). 